\title{
Automatic Spectral Rule-Based Preliminary Mapping of Calibrated Landsat TM and ETM+ Images
}

\author{
Andrea Baraldi, Virginia Puzzolo, Palma Blonda, Lorenzo Bruzzone, Senior Member, IEEE, and Cristina Tarantino
}

\begin{abstract}
Based on purely spectral-domain prior knowledge taken from the remote sensing (RS) literature, an original spectral (fuzzy) rule-based per-pixel classifier is proposed. Requiring no training and supervision to run, the proposed spectral rule-based system is suitable for the preliminary classification (primal sketch, in the Marr sense) of Landsat-5 Thematic Mapper and Landsat-7 Enhanced Thematic Mapper Plus images calibrated into planetary reflectance (albedo) and at-satellite temperature. The classification system consists of a modular hierarchical top-down processing structure, which is adaptive to image statistics, computationally efficient, and easy to modify, augment, or scale to other sensors' spectral properties, like those of the Advanced Spaceborne Thermal Emission and Reflection Radiometer and of the Satellite Pour l'Observation de la Terre (SPOT-4 and -5). As output, the proposed system detects a set of meaningful and reliable fuzzy spectral layers (strata) consistent (in terms of one-to-one or manyto-one relationships) with land cover classes found in levels I and II of the U.S. Geological Survey classification scheme. Although kernel spectral categories (e.g., strong vegetation) are detected without requiring any reference sample, their symbolic meaning is intermediate between those (low) of clusters and segments and those (high) of land cover classes (e.g., forest). This means that the application domain of the kernel spectral strata is by no means alternative to $\mathrm{RS}$ data clustering, image segmentation, and land cover classification. Rather, prior knowledge-based kernel spectral categories are naturally suitable for driving stratified application-specific classification, clustering, or segmentation of RS imagery that could involve training and supervision. The efficacy and robustness of the proposed rule-based system are tested in two operational RS image classification problems.
\end{abstract}

Index Terms-Data clustering, fuzzy rule, fuzzy set (FS), generalization capability, image classification, image color analysis, image segmentation, one-class classifier, prior knowledge, remotely sensed imagery, spectral information, supervised and unsupervised learning from finite data.

\section{INTRODUCTION}

$\mathbf{T}$ HE EXTRACTION of kernel image information layers from multispectral (MS) remote sensing (RS) images is a

Manuscript received July 12, 2005; revised October 3, 2005. This work was originally carried out in the framework of the EU project entitled Landslide Early Warning Integrated Project (LEWIS) under Contract EVG1-CT-200100055 .

A. Baraldi is with the Institute for the Protection and Security of the Citizen, European Commission Joint Research Centre, I-21020 Ispra (Va), Italy (e-mail: andrea.baraldi@jrc.it).

V. Puzzolo is with the Institute for Environmental and Sustainability, I-21020 Ispra (Va), Italy (e-mail: virginia.puzzolo@jrc.it).

P. Blonda and C. Tarantino are with the Institute of Studies on Intelligent Systems for Automation, Italian National Research Council, 70126 Bari, Italy (e-mail: blonda@ba.issia.cnr.it; tarantino@ba.issia.cnr.it).

L. Bruzzone is with the Remote Sensing Laboratory, Department of Information and Communication Technologies, University of Trento, I-38050 Trento, Italy (e-mail: lorenzo.bruzzone@ @ing.unitn.it).

Digital Object Identifier 10.1109/TGRS.2006.874140 well-known problem in the RS literature [1]-[8]. In [1], kernel image information layers are defined as those reliably extracted from RS imagery by means of: 1) domain-specific (e.g., spectral, geometric, textural, semantic, and contextual) prior knowledge and 2) unsupervised (e.g., automatic and data-driven) image processing techniques. This definition implies that kernel image information layers employ no inductive learning-byexample mechanism, i.e., they require no target class sample.

This paper presents an original modular hierarchical topdown spectral rule-based per-pixel classifier capable of detecting a set of kernel spectral (color) layers (strata or categories) in a calibrated Landsat-5 Thematic Mapper (TM) and Landsat7 Enhanced Thematic Mapper Plus (ETM+) satellite imagery. In the proposed system, kernel spectral rules are designed to mimic well-known spectral signatures of target land covers taken from the RS literature. It is important to stress that, on the one hand, the degree of user supervision required to detect spectral rule-based kernel categories is the same as unsupervised data clustering and far inferior to reference sample selection required by supervised classifiers. On the other hand, the symbolic meaning (level of abstraction) of kernel spectral categories (e.g., strong vegetation) is intermediate between those (low) of clusters (say, $n$th cluster) and segments (say, $m$ th segment) and those (high) of land cover (informational) classes (e.g., forest). This implies that the application domain of a kernel spectral category detection system is not an alternative to data clustering, image segmentation, and land cover classification. Rather, all these approaches are complementary in nature. Relevant design and implementation characteristics of the proposed classifier based on prior spectral knowledge are summarized below.

1) Pattern recognition is based exclusively on well-known spectral signatures of target land covers taken from the RS literature and adopted as (fuzzy) data templates. This implies the following.

a) The classification system is pixel-based (contextinsensitive) and purely spectral, i.e., in feature space, it employs no size, shape, location, texture, and semantic information but spectral (color) information exclusively. As a consequence, the proposed mapping system is also computationally efficient.

b) Each pixel data vector consists of an MS (chromatic) data vector component plus a scalar brightness [panchromatic (PAN)] variable.

c) Because no reference data set of examples is assumed to be available, the mapping system employs no supervised data learning mechanism to generate new spectral rules dynamically.

2) The proposed system maps each pixel data vector into a finite set of discrete spectral (color) categories (i.e., types, 
TABLE I

SPECTRAL REgions AND ATMOSPHERIC Windows COMPARED With THE LANDSAT-5 TM, LANDSAT-7 ETM+, SPOT-5 HIGH RESOLUTION GEOMETRIC INSTRUMENT, AND ASTER CHARACTERISTICS [9]. LEGEND-**: FOR LANDSAT-7, BAND 6 IS PROVIDED IN BOTH High AND LOW GAIN, WHICH IS IDENTIFIED AS BANDS TM61 AND TM62, RESPECTIVELY

\begin{tabular}{|c|c|c|c|c|c|c|c|c|}
\hline \multirow{3}{*}{$\begin{array}{c}\text { Spectral } \\
\text { region } \\
\text { name [9] } \\
\text { and } \\
\text { acronym }\end{array}$} & \multirow{3}{*}{$\begin{array}{c}\text { Electro- } \\
\text { magnetic } \\
\text { spectrum } \\
(\mu \mathrm{m})\end{array}$} & \multirow{2}{*}{\multicolumn{2}{|c|}{$\begin{array}{l}\text { Atmospheric } \\
\text { window }\end{array}$}} & \multicolumn{5}{|c|}{$\begin{array}{l}\text { Spectral resolution }(\mu \mathrm{m}) \text { and } \\
\text { spatial resolution }(\mathrm{m})\end{array}$} \\
\hline & & & & \multicolumn{2}{|c|}{ Landsat sensors } & \multicolumn{2}{|c|}{ Spot-5 sensors } & \multirow{2}{*}{$\begin{array}{l}\text { ASTER - sensors } \\
\text { VNIR: band 1-3, } \\
\text { SWIR: band 4-9, } \\
\text { and TIR: band 10- } \\
14 .\end{array}$} \\
\hline & & ID & \begin{tabular}{|l|}
$\begin{array}{l}\text { Spectral } \\
\text { region } \\
(\mu \mathrm{m})\end{array}$ \\
\end{tabular} & $\begin{array}{l}\text { Landsat 5 TM } \\
\text { and } 7 \text { ETM+, } \\
\text { MS }\end{array}$ & \begin{tabular}{|l|} 
Landsat 7 \\
ETM+, \\
PAN \\
\end{tabular} & HRG, MS & $\begin{array}{l}\text { HRG, } \\
\text { PAN }\end{array}$ & \\
\hline $\begin{array}{l}\text { Visible } \\
\text { (Vis, } \\
\text { consisting } \\
\text { of Violet: } \mathrm{V}, \\
\text { Blue: } \mathrm{B}, \\
\text { Green. } \mathrm{G}, \\
\text { Yellow: } Y \text {, } \\
\text { Orange: } \mathrm{O}, \\
\text { Red: R) }\end{array}$ & $\begin{array}{l}0.35-0.72 \\
\text { (V: } 0.35-0.45 . \\
\text { B: } 0.45-0.50 . \\
\text { G: } 0.50-0.57 \\
\text { Y: } 0.57-0.60 \\
\text { O: } 0.60-0.65 \\
\text { R: } 0.65-0.72 \text { ) }\end{array}$ & 1 & $0.3-1.3$ & $\begin{array}{l}\text { 1-B: } 0.45-0.52 . \\
\text { 2-G: } 0.52-0.60 . \\
\text { 3-R: } 0.63-0.69 . \\
30 \mathrm{~m}\end{array}$ & $\begin{array}{l}0.52-0.90 . \\
15 \mathrm{~m}\end{array}$ & $\begin{array}{l}\text { I-G: } 0.50- \\
0.59 . \\
2-\mathrm{R}: 0.61- \\
0.68 . \\
\\
10 \mathrm{~m}\end{array}$ & $\begin{array}{l}0.48-0.71 . \\
5 \mathrm{~m}\end{array}$ & $\begin{array}{l}\text { 1-G: } 0.52-0.60 . \\
\text { 2-R: } 0.63-0.69 . \\
15 \mathrm{~m}\end{array}$ \\
\hline $\begin{array}{l}\text { Near } \\
\text { Infrared } \\
\text { (NIR) }\end{array}$ & $0.72-1.3$ & & & $\begin{array}{l}\text { 4-NIR: } 0.76- \\
0.90 . \\
30 \mathrm{~m}\end{array}$ & & $\begin{array}{l}\text { 3-NIR: } 0.79- \\
0.89 . \\
10 \mathrm{~m}\end{array}$ & & $\begin{array}{l}\text { 3-NIR: } 0.76-0.86 . \\
15 \mathrm{~m}\end{array}$ \\
\hline \multirow{3}{*}{$\begin{array}{l}\text { Middle IR } \\
\text { (MIR) }\end{array}$} & \multirow[b]{2}{*}{$1.3-3.0$} & 2 & $1.5-1.8$ & $\begin{array}{l}\text { 5-MIR1: } 1.55- \\
1.75 . \\
30 . \mathrm{m}\end{array}$ & & $\begin{array}{l}\text { 4-MIR: } 1.58- \\
1.75 . \\
20 \mathrm{~m}\end{array}$ & & $\begin{array}{l}\text { 4-MIR1: 1.600-1.700. } \\
30 \mathrm{~m}\end{array}$ \\
\hline & & 3 & $2.0-2.6$ & $\begin{array}{l}\text { 7-MIR2: } 2.08- \\
2.35 . \\
30 \mathrm{~m}\end{array}$ & & & & $\begin{array}{l}\text { 5-MIR2: 2.145-2.185. } \\
\text { 6-MIR3: 2.185-2.225. } \\
\text { 7-MIR4: } 2.235-2.285 \\
\text { 8-MIR1: } 2.295-2.365 \\
\text { 9-MIR6: } 2.360-2.430 . \\
\text { 30m }\end{array}$ \\
\hline & $3.0-7.0$ & $\frac{4}{5}$ & $\begin{array}{l}3.0-3.6 \\
42-5\end{array}$ & & & & & \\
\hline Far IR (FIR) & $\begin{array}{l}7.0-15.0, \\
\text { including } \\
\text { Thermal IR } \\
\text { (TIR): } 8.0- \\
12.0\end{array}$ & 6 & $7.0-15.0$ & $\begin{array}{l}\text { 6-TIR*: } 10.4- \\
12.5 \text {. } \\
\text { TM 5: } 120 \mathrm{~m} \\
\text { ETM+7:60m }\end{array}$ & & & & $\begin{array}{l}\text { 10-TIR1: 8.125- } \\
\text { 8.475. } \\
\text { 11-TR2: 8.475- } \\
\text { 8.825. } \\
\text { 12-TIR3: 8.925- } \\
\text { 9.275. } \\
\text { 13-TIR4: } 10.25- \\
\text { 10.995. } \\
\text { 14-TIR5: 10.95- } \\
\text { 11.65. } \\
\text { 90m }\end{array}$ \\
\hline
\end{tabular}

labels, and strata), which are called kernel spectral types or spectral candidate areas.

3) To detect kernel spectral categories (fuzzy) decision rules, combine several sources of (either crisp or fuzzy, depending on the implementation) spectral evidence. In particular:

a) Each (fuzzy) decision rule is a logical expression of fuzzy sets (FSs).

b) Each (fuzzy) decision rule is designed to mimic a well-known spectral signature of a target land cover in different portions of the electromagnetic spectrum.

c) FSs provide a complete grid partition of the purely spectral feature space.

4) FSs are adaptive to image statistics, i.e., the proposed system of rules employs unsupervised data learning mechanisms.

5) In the proposed implementation, kernel spectral categories are:

a) conceived for Landsat-5 TM and 7 ETM+ imagery calibrated into planetary reflectance (albedo) and atsatellite temperature [10];

b) scalable to other existing medium- to high-resolution spaceborne optical sensors sensitive to MS and PAN portions of the electromagnetic spectrum, such as the Advanced Spaceborne Thermal Emission and Reflection Radiometer (ASTER) and Systeme Pour l'Observation de la Terre (SPOT-4 and SPOT-5) (refer to Table I, where some acronyms adopted in the rest of this paper are introduced, and see Fig. 1);

c) capable of replacing and/or extending the set of kernel categories implemented in the alternative rule-based

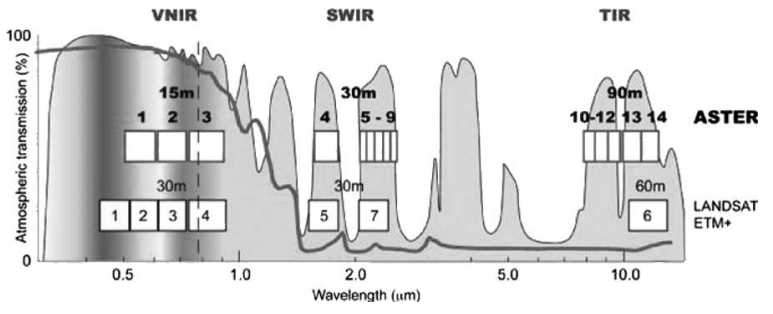

Fig. 1. Spectral resolution characteristics of ASTER, which is mounted onboard the Terra platform, in comparison with atmospheric windows and Landsat-7 ETM+ spectral resolution characteristics (downloaded from [12]). The ASTER instrument consists of three separate instrument subsystems, namely: 1) visible and near infrared (VNIR); 2) shortwave infrared (SWIR); and the 3 ) thermal infrared (TIR).

approaches, such as [1] (namely, water, roads, vegetation, nonvegetation, and ambiguous);

d) mutually exclusive and totally exhaustive [11];

e) consistent (in terms of one-to-one or many-to-one relationships) with the set of land cover classes adopted by levels I and II of the U.S. Geological Survey (USGS) land cover classification scheme (refer to Fig. 2, where the land cover hierarchy adopted in this paper is depicted).

6) The output product of the proposed image mapping system is a discrete map consisting of kernel spectral strata. As already observed by Nagao and Matsuyama [2, p. 30], this output map is equivalent to a primal sketch or a preliminary map. In the Marr sense, a primal sketch of an input image "explicitly reveals information about geometrical distribution and organization of color (intensity) changes" $\left[12\right.$, p. 37]. ${ }^{1}$

The proposed kernel spectral category detection system can be considered of current interest to a large segment of RS readership owing to its potential application domain, which is threefold.

1) In recent years, the launch of (very) high resolution (VHR) spaceborne MS scanners has meant that, for many applications, purely supervised analysis is no longer feasible. Surface areas to be analyzed are large and heterogeneous, and it is often impractical, impossible, or too expensive to collect sufficient training samples to estimate the statistics of every target class within the area of interest. To overcome the limitations of purely supervised classification of an RS imagery, two-stage hybrid learning systems are recommended [2], [9, p. 185], [14]. In a hybrid data learning framework, a twostage cascade of the proposed spectral knowledge-based first stage with stratified image enhancement, clustering,

\footnotetext{
${ }^{1}$ Marr, who stood against the incorporation of syntactic and semantic knowledge about the structure of a scene into the early stages of visual processing [13], proposes a famous representational framework for deriving shape information from images. It consists of a hierarchy of a primal sketch (or preliminary map), a two-and-a-half-dimensional (2.5-D) sketch, and a three-dimensional (3-D) model representation. In recent years, a widely accepted interpretation of the image understanding problem conceives the high-level semantic processing stage as provided with feedback mechanisms capable of passing on to the lowlevel signal processing stage some goal-oriented symbolic information, e.g., information based on semantic and contextual (neighboring) rules, which are useful in refining the initial (general-purpose) segmentation [1], [2] .
} 


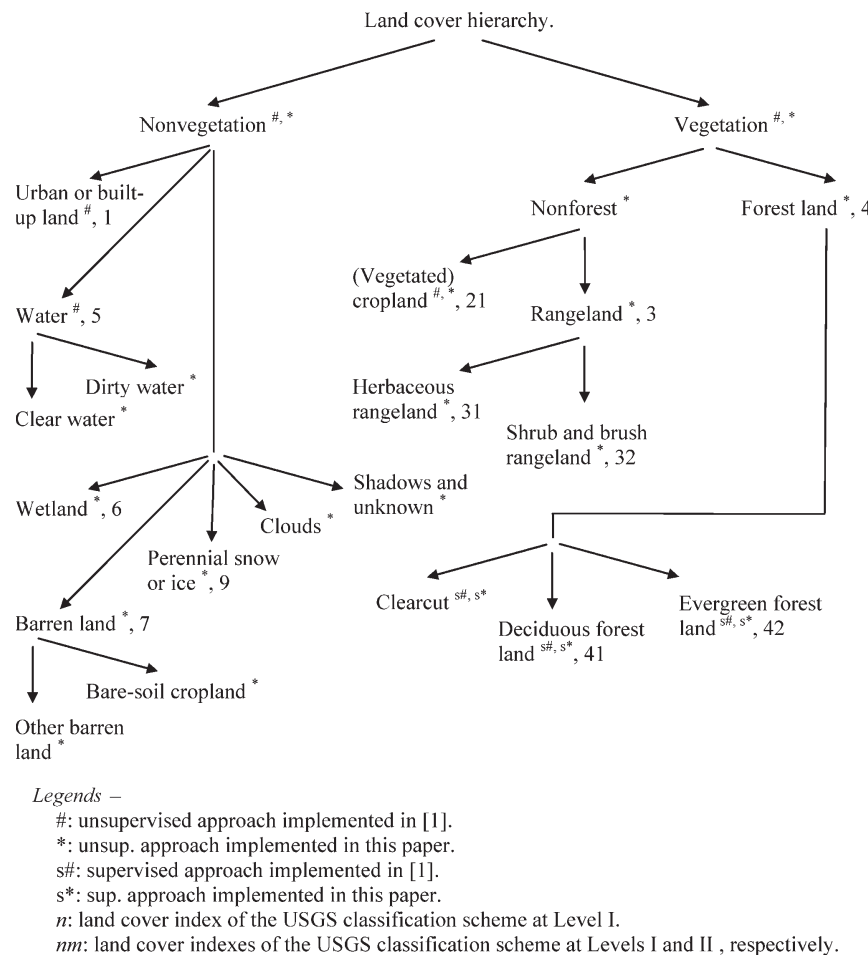

Fig. 2. Land cover hierarchy adopted in this work. Kernel spectral categories (i.e., types, layers, and strata) detected by the proposed spectral knowledgebased system are related to land cover (informational) classes in terms of oneto-one or many-to-one relationships (refer to text).

segmentation, or classification second-stage algorithms is straightforward. In particular:

a) A kernel spectral map can facilitate the work of a human photointepreter (supervisor) in locating and selecting regions of interest (ROIs) in an input RS picture provided with little or no ground truth information. Selected ROIs can provide target class examples to either plug-in (e.g., maximum likelihood) or inductive learning classifiers [e.g., multilayer perceptron (MLP)] [15], [16].

b) Some authors strongly discourage exploitation of state-of-the-art RS image topographic correction methods where a single non-Lambertian surface reflectance model (in which the reflectance depends on both observation and incident angles) is applied image-wide, although this model depends on surface roughness and is class-specific. To overcome this problem, the land cover type of each pixel should be known in advance [17]. If a kernel spectral map is generated from an RS image before a topographic normalization takes place, it is important to remember that a single stratum of the kernel spectral map may gather pixels belonging to two or more land cover classes as their differences in class-specific spectral signatures are counterbalanced by changes in illumination conditions (e.g., coniferous forests in illuminated areas and deciduous forests located in shadow areas are likely to be captured by the same kernel spectral layer). In this context, multiple independent stratified (i.e., layer-specific) topographic corrections (each one is potentially capable of exploiting an ad hoc non-Lambertian behavior) seem better suited for compensating differences in solar illumination due to the irregular shape of the terrain. In other words, within each spectral map layer, similar planetary reflectance values are expected to be easier to project onto separate portions of a flat-normalized reflectance space due to differences in class labels exclusively.

c) First-stage kernel spectral strata detection can reduce the image domain subjected to second-stage clustering/segmentation/classification algorithms. In particular:

i) Stratified clustering or data quantization ${ }^{2}$ or principal component analysis makes the unsupervised data analysis problem easier to solve. For example, in [1], boundaries of water regions are improved with a clustering technique.

ii) Stratified segmentation (also refer to Section IIIG3 below) makes the image segmentation problem easier to solve. In general, it is well known that general-purpose segmentation is less effective than category-oriented knowledge-driven segmentation [1].

iii) Stratified classification (also refer to Section IIIG2 below) makes the supervised data classification problem easier to solve. For example, in [1], the vegetation spectral category detected by a spectral rule-based system is further partitioned into clearcut, deciduous, and evergreen forest types by an induced classifier.

2) A kernel spectral map can be employed to mask out clouds and snow areas from an input raw image. For example, in an unsupervised change detector [18], a pair of cloud-free input scenes of the same area is required.

3) A mutually exclusive and totally exhaustive preliminary map can provide a quick-look type of classification product (baseline map) capable of characterizing the quality of pictures in RS image databases. This may be useful in:

a) minimizing purchased risks resulting from cloudy weather (traditionally, usability of raw picture data is parameterized by a synthetic visibility ratio) as well as undesired land surface phenomena (e.g. flooding and drought);

b) querying a content-based image database retrieval system;

c) providing an expert photointerpreter with a preliminary spectral map more reliable (it simultaneously exploits all spectral bands at hand) and more accurate than the traditional photointerpretation of an unlabeled false-color raw image composition in a 3-D color space [19].

The rest of this paper is organized as follows. In Section II, keywords and definitions are introduced. In Section III, the novel spectral rule-based system is proposed. In Section IV, to highlight its degree of novelty, the proposed approach is compared against the related rule-based classifiers presented

\footnotetext{
${ }^{2}$ As (predictive) vector quantizers are also used for (non predictive) data clustering [21, p. 177] a typical choice for data clustering is to employ a vector quantization algorithm (i.e., one capable of minimizing a mean square error), such as the standard Hard C-Means [22].
} 
in the RS literature in recent years. In Section V, experimental results are presented and discussed. In Section VI, conclusions are reported.

\section{KEYWORDS AND DEFINITIONS}

Inspired by Marr's works in computer vision [13], the central concept of primal sketch or preliminary map of an MS image, which is introduced in Section I and adopted in the rest of this paper, is defined as follows. "A preliminary map of an MS image consists of a discrete set of strata (layers, types, either mutually exclusive or overlapping in part) providing explicit information about the spatial distribution of pixels featuring similarities in multispectral values before any supervised learning-from-data analysis takes place." This definition yields two important corollaries.

1) By definition, this preliminary mapping is generated from either learning-by-rule systems, which are based on a priori knowledge, or unsupervised learning-by-example methods [20], [21]. As a consequence, the output product of a traditional data clustering or image segmentation algorithm falls in the class of preliminary maps or primal sketches of an input MS image [22]-[26]. Vice versa, the output product of a supervised classifier (e.g., a plug-in maximum-likelihood classifier and a supervised inductive learning MLP), which is known as classification (i.e., thematic and informational) map consisting of land cover classes, is not a preliminary map of an input MS image.

2) By definition, this preliminary mapping is independent of the symbolic meaning associated with the spectral strata, which may be equal to 0 . For example, neither data clustering nor general-purpose image segmentation algorithms provide their informational primitives (clusters and segments, respectively) with any symbolic (semantic) meaning (e.g., in general, clusters and segments are identified by numbers).

A taxonomy of the several sources of a priori knowledge capable of providing information useful in generating a preliminary map of an RS image can be as follows [2]:

1) prior knowledge about acquisition conditions (e.g., date, altitude, weather conditions, and sun elevation);

2) prior knowledge about the target surface area (e.g., reference maps and ground survey sample data);

3) prior knowledge about the intrinsic attributes of a target object such as:

a) geometrical/spatial attributes, e.g., shape, size, orientation, and location;

b) topological attributes, e.g., connectivity;

c) spectral (chromatic) properties (this is the kind of a priori knowledge that is extracted from RS literature and employed in this paper);

d) PAN (chromatic) properties;

e) textural properties [27].

These attributes can be employed in the light of the compactness hypothesis, which is the basis for object recognition [28]. It states that similar objects are close in their representations.

4) prior knowledge about topological (e.g., adjacency, inclusion, and intersection), contextual (neighboring), and/or semantic relationships (constraints) among image ob- jects. For example, a small vegetation segment surrounded by a built-up area in the preliminary map can be further recognized as belonging to an urban context in the land cover map generated by the high-level processing stage [1].

In the traditional field of artificial intelligence [20], [21], prior knowledge-based systems of decision rules consist of a set of simultaneous premises (conditions on scalar input variables) and an output consequence (action), e.g.,

IF (temperature is low) and (pressure is high)

THEN (ignition value is 0.7 ).

Let us identify premises with $A_{r, n}, r=1, \ldots, R$, where $R$ is the number of rules, with $n=1, \ldots, N$, where $N$ is the input space dimensionality, and consequences as $B_{r, m}$, $r=1, \ldots, R, m \in\{1, M\}$, where $M$ is the output space dimensionality. Let us focus our attention on rules that combine premises by logical AND operators. Thus, a decision rule becomes

$$
\operatorname{IF}\left(\bigcap_{n=1}^{N} A_{r, n}\right) \operatorname{THEN}\left(B_{r, m}\right) .
$$

By definition, an FS is an ordered pair $\mathrm{FS}\left(x_{n}\right)_{L}=$ $\left\{\left(x_{n}, \mu_{L}\left(x_{n}\right) \mid x_{n} \in \mathfrak{R}, \quad n \in\{1, N\}\right\}\right.$, where a membership function (MF) $\mu_{L}\left(x_{n}\right)$, which is associated with a (e.g., linguistic) label $L$, maps the scalar input space $\mathfrak{R}$ to the bounded nonnegative real membership space $[0,1]$. In this case, the IF-THEN rule is termed fuzzy [29]. If a membership space contains only two points, i.e., 0 and 1 , then the decision is not fuzzy. In this case, it is instead termed hard or crisp. A typical choice of a fuzzy MF is the one-dimensional (1-D) Gaussian function.

By definition, given an $N$-dimensional feature space, the IF part of a fuzzy rule consists of a logical AND combination of one up to $N$ 1-D FSs, which is equivalent to one $N$-dimensional rule patch (activation domain), i.e., there is one rule patch per fuzzy rule [see Fig. 3(a) and (b)] [30], [31].

\section{PRoposed ApProACH}

This section aims at providing the reader with sufficient information for the implementation to be reproduced. In particular, it describes the implemented spectral rule-based Landsat TM and ETM+ image mapping system in terms of:

1) feature extraction;

2) automatic valley detection in a bimodal distribution to implement feature space adaptive grid partitioning;

3) feature space adaptive grid partitioning, i.e., FS computation in feature space;

4) rule-based system architecture, which is sketched in Fig. 4 as a top-down prior knowledge-based classifier consisting of a first- and second-level processing of kernel spectral categories. In particular:

a) The first-level computation of kernel spectral types consists of:

i) Kernel spectral (fuzzy) rules, which are generated from spectral reflectance curves extracted from the RS literature (see Fig. 5) and partitioned into 


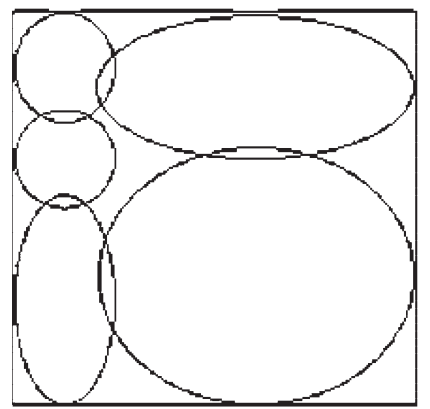

(a)

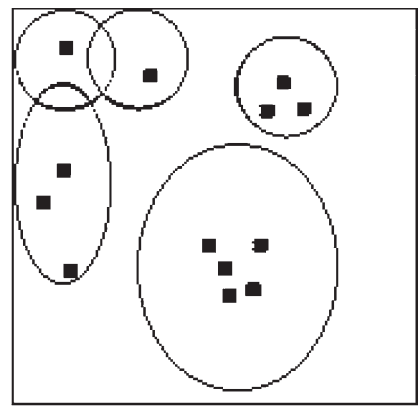

(b)
Fig. 3. (a) Example of irregular but complete grid partitioning of a 2-D feature space provided by a fuzzy rule-based system whose number of fuzzy rules (rule patches) is five. The rule-based classifier proposed in this paper pursues irregular but complete grid partitioning. It employs a fixed (applicationindependent) number of rules, whereas the size of rule patches (activation domain) is data-driven. (b) Example of scatter partitioning of a 2-D input space, where the number and location of the IF parts of the fuzzy rules (rule patches) are adaptive (data-driven, problem-specific). In this case, there are five fuzzy rules whose rule patches cover a selected portion of input space where input data samples, which are represented as black dots, are likely to fall.

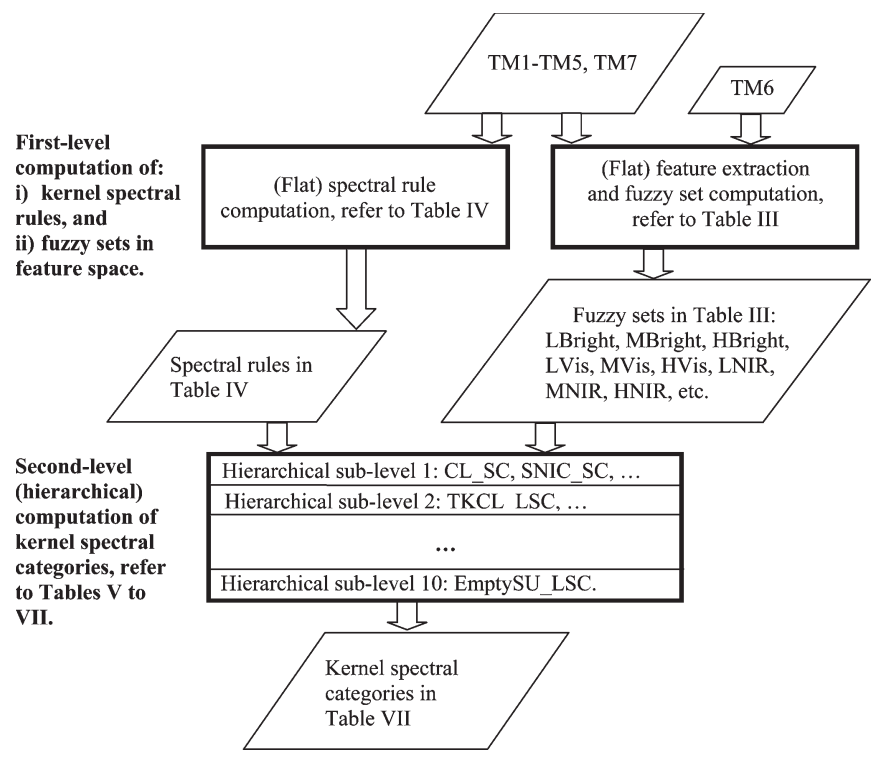

Fig. 4. Two-stage spectral rule-based system architecture. Landsat bands TM1-TM5 and TM7 are meant to be calibrated into planetary reflectance values (albedo), band TM6 into at-satellite temperature.

different portions of the electromagnetic spectrum. Kernel (fuzzy) spectral rules are implemented as logical expressions of scalar (numerical) variables combined with relational operators (e.g., $\geq$ ) and logical operators (e.g., logical AND). Scalar variables are the Landsat bands TM1-TM5 and TM7 calibrated into planetary reflectance values (refer to acronyms adopted in Table I). It is noteworthy that band TM6, which is calibrated into at-satellite temperature values, is not considered at this stage [10].

ii) Feature extraction and FS computation starting from a calibrated TM1-TM7 feature space.

The first-level processing is flat, i.e., nonhierarchical, as spectral rules and fuzzy sets can be computed in parallel. Spectral rules are listed explicitly in this paper to allow implementation to be reproduced.

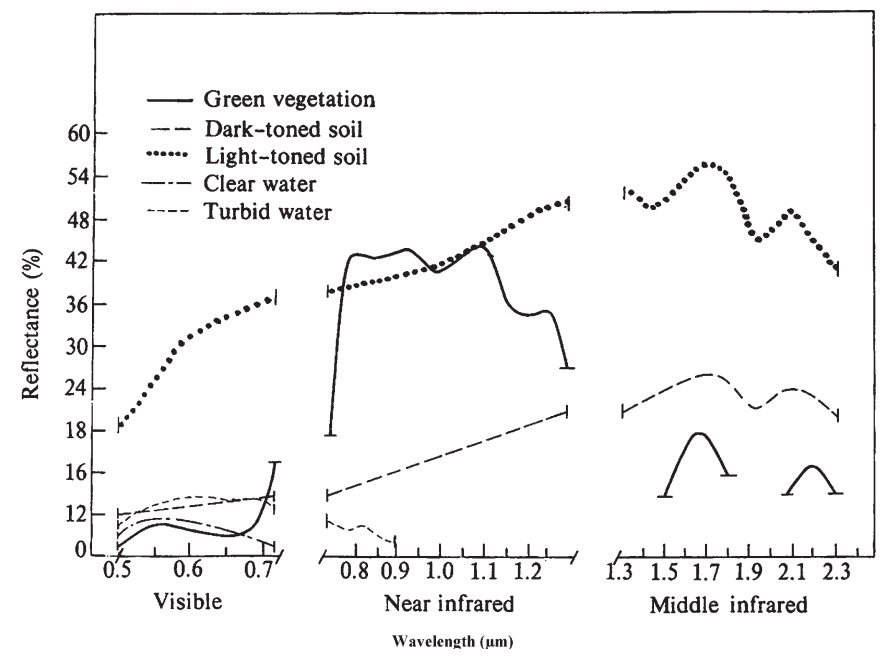

Fig. 5. Spectral reflectance curves for green vegetation, light and dark soils, and clear and turbid water (taken from [9, p. 276]).

b) Unlike the flat first-level processing, the second-level processing of kernel spectral categories consists of a hierarchy of logical expressions of binary variables. These binary variables are the outputs of spectral rules and FSs computed at first-level processing. Logical operators (e.g., logical AND) are employed at this stage exclusively. Sixty-seven spectral categories are listed explicitly in this paper to allow implementation to be reproduced.

5) cascade of kernel spectral category detection with stratified (i.e., category-, class-, or application domainspecific) classification, clustering, or segmentation.

For the sake of simplicity (e.g., to reduce the computation time of an operational rule-based system), crisp spectral rules (i.e., rules whose membership space is binary, refer to Section II) rather than fuzzy spectral rules are proposed hereafter. It is noteworthy that a fuzzy extension of a crisp decision rule set is straightforward, by way of commercial image processing software toolboxes, by means of the following [32]-[34].

1) Input feature normalization step, e.g., implemented as a mathematical $S$-MF [32] $\mu s_{n}\left(x_{n}\right)=S\left(x_{n}\right.$, $\left.\operatorname{Min}_{n}, \operatorname{Max}_{n}\right) \in[0,1]$, which is controlled by a pair of feature-dependent bandwidth parameters $\operatorname{Min}_{n}$ and $\operatorname{Max}_{n}$ (refer further to Table III), with input variable $x_{n} \in X=\left\{x_{1}, \ldots, x_{N}\right\}, X \subseteq \mathfrak{R}^{N}$, where $X$ is an $N$-dimensional input space of real values (also refer to Section II). This input feature normalization step transforms a measurement space, which is, in general, unbounded and provided with nonhomogeneous physical units, into a homogeneous membership space equivalent to a unit hypercube.

2) A fuzzy logical OR operator by maximum, which is identified as $\cup$, to replace any binary logical OR operator.

3) A fuzzy logical AND operator by minimum, which is identified as $\cap$, to replace any binary logical and operator.

4) A mathematical $Z$-MF $\mu z_{i}\left(x_{i}\right)=1-S\left(x_{i}, \operatorname{Min}_{i}, \operatorname{Max}_{i}\right)$, $\mu z_{i}\left(x_{i}\right) \in[0,1]$ equivalent to the inverse $S$-MF transform, in place of any binary logical NOT operator. 


\section{A. Feature Extraction}

Input features (also called spectral data primitives or elementary spectral bands) of the proposed rule-based RS image mapping system are the seven bands of the Landsat imagery, i.e., TM1-TM7 (refer to Table I and Fig. 4), which is calibrated into planetary reflectance values. Spectral data primitives TM1-TM7 are employed to extract seven spectral features capable of capturing image-independent properties of the spectral signatures of the target land cover classes. In particular, extracted features are computed as either linear combinations of elementary spectral bands or ratios between spectral data primitives acquired in different (in general, adjacent) portions of the electromagnetic spectrum (where band ratios provide a discrete approximation of estimating the first derivative of a continuous spectral signature at different positions across the electromagnetic wavelength domain). The extracted spectral features are listed hereinafter (also refer to Table I).

1) Brightness (Bright): Perceived luminance [35]. In line with [2], it is computed as a weighted combination of bands TM1-TM5 and TM7.

2) Visible (Vis) reflectance is the estimated reflectance in the visible portion of the electromagnetic spectrum. It linearly combines bands TM1-TM3 that are individually unfeasible for being employed in land cover discrimination due to their typically small range and high correlation.

3) Near-infrared (NIR) reflectance is the estimated reflectance in the NIR portion of the electromagnetic spectrum.

4) Middle infrared (MIR) reflectance is the estimated reflectance in the MIR portion of the electromagnetic spectrum.

5) Thermal infrared (IR) reflectance is the estimated reflectance in the TIR portion of the electromagnetic spectrum.

6) Normalized difference vegetation index (NDVI), which is aimed at reducing MS measurements to a single value for predicting and assessing vegetation characteristics such as species, leaf area, stress, and biomass. It should be insensitive to shadow areas [2], [36].

7) Normalized difference bare soil index (NDBSI), which is aimed at enhancing bare soil areas, fallow lands, and vegetation with marked background response. This single value should be useful for predicting and assessing bare soil characteristics such as roughness, moisture content, amount of organic matter, and relative percentages of clay, silt, and sand [37].

8) Normalized difference snow index (NDSI), which is aimed at discriminating snow/ice from all remaining surface classes, including clouds and cold and highly reflective barren land [38].

9) Band MIR/TIR composite (MIRTIR), which is aimed at mitigating well-known difficulties in separating thin and warm clouds from ice areas and cold and highly reflective barren land [38].

1) Estimated Reflectance Variables Bright, Vis, NIR, MIR, TIR, and MIRTIR: Because atmospheric (Rayleigh) scattering of light decreases with wavelength, gray level differences between shadow and nonshadow areas become especially relevant in the R, NIR, and MIR bands. To enhance this difference, the brightness expression, adapted from [2], is the weighted average of the calibrated TM data bands 1-5 (TM1-TM5) and 7 (TM7), i.e.,

$$
\begin{array}{r}
\text { Bright }=(1 / 8) *(\mathrm{TM} 1+\mathrm{TM} 2+2 . * \mathrm{TM} 3+2 . * \mathrm{TM} 4 \\
+\mathrm{TM} 5+\mathrm{TM} 7), \quad \text { Bright } \in[0,1] .
\end{array}
$$

Feature Vis combines calibrated TM data bands 1-3 (TM1-TM3) as the average of channels R, G, and B, i.e.,

$$
\text { Vis }=(1 / 3) *(\text { TM1 }+ \text { TM2 }+ \text { TM } 3), \quad \text { Vis } \in[0,1] .
$$

Feature NIR is equivalent to the calibrated TM channel 4 (TM4), i.e.,

$$
\mathrm{NIR}=\mathrm{TM} 4, \quad \mathrm{NIR} \in[0,1] .
$$

Feature MIR1 and MIR2 are equivalent to the calibrated TM channels 5 (TM5) and 7 (TM7), respectively, i.e.,

$$
\text { MIR1 }=\text { TM5 }, \quad \text { MIR1 } \in[0,1]
$$

and

$$
\text { MIR2 }=\text { TM7, } \quad \text { MIR2 } \in[0,1] .
$$

Although they both belong to the MIR portion of the electromagnetic spectrum (refer to Table I) and tend to be strongly correlated, channels TM5 and TM7 are dealt with separately. In fact, despite their high correlation and similar variance, their means are quite different. For example, in a test image adopted in the experimental session (see Fig. 8 in Section V), Corr(TM5, TM7) $=0.96$, where Mean(TM5) $=$ 44.6, $\operatorname{StanDev}(\mathrm{TM} 5)=19.8$, whereas $\operatorname{Mean}(\mathrm{TM} 7)=27.8$, $\operatorname{StanDev}(\mathrm{TM} 7)=19.0$. In general, clouds and snow/ice are highly reflective in any portion of the electromagnetic spectrum, except in MIR, where snow is slightly reflective.

Feature TIR is currently computed as the calibrated low-gain TM channel 6-2 (TM62) in Kelvin degrees, i.e.,

$$
\mathrm{TIR}=\mathrm{TM} 62, \quad \text { TIR } \geq 0 \mathrm{~K} .
$$

In general, clouds tend to be colder (e.g., below $300 \mathrm{~K}$; refer to Section III-C) and feature higher reflectance at the 1700-nm wavelength (equivalent to channel TM5; refer to Table I) than cold and highly reflective barren land. To enhance this difference in spectral behaviors, a well-known TM band 5/6 composite specifically developed for cloud detection is [38]

$$
\text { MIRTIR }=(1-\text { MIR1 }) * \text { TIR, } \quad \text { MIRTIR } \geq 0 .
$$

2) Surface Type Indexes NDVI, NDBSI, and NDSI: To exploit the differences in the reflectance patterns of green vegetation from other objects' spectral signatures, vegetation indexes are based on ratios or linear combinations of spectral responses in specific portions of the electromagnetic spectrum. For example, a well-known NDVI is computed from TM data bands 3 (TM3) and 4 (TM4) as [36]

$$
\begin{array}{r}
\mathrm{NDVI}=(\mathrm{TM} 4-\mathrm{TM} 3) /(\mathrm{TM} 4+\mathrm{TM} 3+0.001) \\
\text { NDVI } \in[-1,1] .
\end{array}
$$


Important properties of NDVI are the following.

1) Within the same leaf-on (leaf-off) season, NDVI is scarcely affected by the time of acquisition.

2) In line with the ratio vegetation index RVI = TM4/TM3, which is adopted in [2], NDVI is useful in extracting vegetation regions even when they are in shadow.

3) NDVI per se is unable to highlight subtle differences in canopy density [37]. Based on our experience, condition NDVI $>0.35$ is a strong (necessary, but not sufficient) indication of the presence of vegetated areas.

4) According to [36], where two indexes are considered equivalent if the decision made on the basis of one index could have been made equally well on the basis of the other index, NDVI is equivalent to NDVI and to the transformed vegetation index $\mathrm{TVI}=(\mathrm{NDVI}+0.5)^{1 / 2}$ for decision making. In contrast with [2], we prefer NDVI to RVI due to its finite range of change, which makes it more intuitive to use, and to its many references in the existing literature.

Similar to the concept of NDVI, the original NDBSI proposed in this paper is computed from TM data bands 4 (TM4) and 5 (TM5) according to the following equation:

$$
\begin{aligned}
\mathrm{NDBSI}=(\mathrm{TM} 5-\mathrm{TM} 4) /(\mathrm{TM} 5+\mathrm{TM} 4+0.001), & \\
& \text { NDBSI } \in[-1,1] .
\end{aligned}
$$

In general, condition NDBSI $>-0.20$ is a strong (necessary, but not sufficient) indication of the presence of bare soil areas. As far as our knowledge goes, this bare soil index is an original adaptation of the normalized difference bare soil index "BIO" found in [37] and is defined as

$$
\begin{array}{r}
\mathrm{BIO}=[(\mathrm{TM} 5+\mathrm{TM} 3)-(\mathrm{TM} 4+\mathrm{TM} 1)] /[(\mathrm{TM} 5+\mathrm{TM} 3) \\
+(\mathrm{TM} 4+\mathrm{TM} 1)], \quad \text { BIO } \in[-1,1] .
\end{array}
$$

It is noteworthy that in a test image adopted in the experimental session (see Fig. 8 in Section V), standardized versions of variables NDBSI, NDVI, and BIO (i.e., standard variables featuring zero mean and unit variance, which makes their dynamics comparable) provide the following statistics: Corr(NDBSI, NDVI) $=-0.56$. First eigenvalue $($ NDBSI, NDVI) $=75 \%$ of the total variance (equal to the sum of the main diagonal of the covariance matrix). Corr(BIO, NDVI $)=-0.69$. First eigenvalue(NDBSI, NDVI) $=84 \%$ of the total variance.

These results are consistent with a theoretical analysis of (10)-(12). They show that NDBSI and NDVI are inversely but less correlated than BIO and NDVI.

Features NDVI and NDBSI provide two estimates (i.e., ratios and derivatives) of the spectral phenomena occurring in different portions of the electromagnetic spectrum. In our experiments (refer to Section $\mathrm{V}$ below), these two estimates provide complementary sources of evidence for separating vegetation from nonvegetation kernel categories with a high level of confidence, e.g., if [(NDVI is high) and (NDBSI is low)] then vegetation is very likely to occur.

To enhance the difference between the typical spectral signatures of snow/ice from other objects' spectral signatures
TABLE II

TASSElED CAP COEFFICIENTS FOR LANDSAT-7 ETM+ PlanetaRY REFLECTANCE (TAKEN FROM [40])

\begin{tabular}{|l|l|l|l|l|l|l|}
\hline & TM1 & TM2 & TM3 & TM4 & TM5 & TM7 \\
\hline Brightness & 0.3561 & 0.3972 & 0.3904 & 0.6966 & 0.2286 & 0.1596 \\
\hline Greenness & -0.3344 & -0.3544 & -0.4556 & 0.6966 & -0.0242 & -0.2630 \\
\hline Wetness & 0.2626 & 0.2141 & 0.0926 & 0.0656 & -0.7629 & -0.5388 \\
\hline
\end{tabular}

(including clouds and bare soil), a well-known normalized difference snow index is [38]

$$
\begin{aligned}
& \text { NDSI }=(\text { TM } 2-\text { TM5 }) /(\text { TM } 2+\text { TM } 5+0.001) \\
& \text { NDSI } \in[-1,1] .
\end{aligned}
$$

This NDSI expression exploits the peculiar property of snow being (in general) brighter than the vegetation and bare soil in the visible portion of the electromagnetic spectrum and (much) darker than the clouds at the 1700-nm wavelength (equivalent to channel TM5; refer to Table I). Starting from this prior spectral knowledge, our system replaces band TM2 in (13) with feature Vis [see (4)]. Thus, (13) becomes

$$
\begin{aligned}
\mathrm{NDSI}=(\mathrm{Vis}-\mathrm{TM} 5) /(\mathrm{Vis}+\mathrm{TM} 5+ & 0.001) \\
& \text { NDSI } \in[-1,1] .
\end{aligned}
$$

Based on our experience, condition NDSI $>0.50$ is a strong (necessary, but not sufficient) indication of the presence of snow areas.

Suitable for detecting built-up areas, a normalized difference index accounting for the blue-band component in built-up areas and barren land (NDBBBI) may be defined as

$$
\begin{aligned}
\mathrm{NDBBBI}=(\mathrm{TM} 1-\mathrm{TM} 5) /(\mathrm{TM} 1+\mathrm{TM} 5+0.001) & \\
& \text { NDBBBI } \in[-1,1]
\end{aligned}
$$

which would be highly correlated with NDSI. Thus, in our system, NDBBBI = NDSI.

It is noteworthy that extraction of features NDVI, NDBSI, and NDSI based on (10), (11), and (14) may also hold for SPOT-5 HRG and ASTER images (refer to Table I). On the contrary, features NDBSI and NDSI cannot be extracted from a very high spatial resolution satellite imagery such as Quickbird and Ikonos, where no spectral band is acquired in the MIR portion of the electromagnetic spectrum.

To summarize, the proposed rule-based classification of Landsat imagery is expected to lose much of its efficacy in partitioning vegetation from nonvegetation kernel categories when it is (down-)scaled to the spectral resolution characterizing VHR optical images like those of Quickbird and Ikonos. This theoretical expectation is confirmed by some preliminary experiments conducted on Ikonos imagery by the authors of this paper. It is also supported by experimental results gathered from the existing literature (e.g., in [39], coca plantations in tropical forest areas can be directly identified in single-date SPOT-4 imagery, but cannot be directly detected in single-date VHR pan-sharpened four-band Ikonos imagery).

3) Relationships Between Extracted Features and Tasseled Cap Transformation: It is noteworthy that the set of extracted features Bright, NDVI, and NDSI appear to be highly correlated to the first three components, which are termed brightness, 


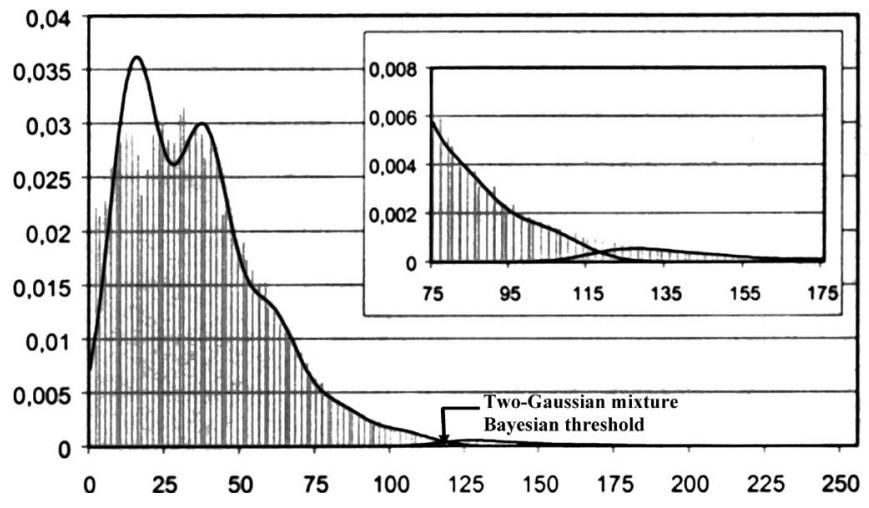

Fig. 6. Typical bimodal distribution, featuring a large peak and a long tail (taken from [18]).

greenness, and wetness, of a tasseled cap transformation based on Landsat-7 images calibrated into planetary reflectance values (refer to Table II) [40]. In the work by Huang et al., the first three tasseled cap transformation components account for over $97 \%$ of the cumulative spectral variance of the individual scenes employed to optimize the tasseled cap transformation coefficients.

Further evidence of the conceptual relationship between the aforementioned features Bright and NDVI with the first two components of a tasseled cap transformation for very high spatial resolution Ikonos MS images (where no MIR channel exists) can be found in [41]. In this work by Horne, the first two components account for over $98 \%$ of the spectral variance of the individual scenes employed to estimate the transformation coefficients.

To summarize, based on the existing literature dealing with tasseled cap transformations of RS images acquired from different optical sensors, the aforementioned features Bright, NDVI, and NDSI appear to provide an effective spectral space dimensionality reduction irrespective (to some degree) of the sensor at hand. However, as one of its original contributions (see succeeding sections), this paper points out that the detection of kernel spectral strata in spaceborne MS imagery becomes more effective when features Bright, NDVI, and NDSI are combined with additional features such as NDBSI, Vis, NIR, MIR1, MIR2, TIR, and MIRTIR (defined in Sections III-A1 and III-A2).

\section{B. Automatic Valley Detection in a Bimodal Distribution}

In the proposed rule-based classifier, the input feature space is grid-partitioned into FSs whose activation domains (rule patches) are data-driven (i.e., FSs are adaptive to image statistics) [see Fig. 3(a)]. In particular, the objective of this section is to investigate an unsupervised data learning (automatic) procedure capable of modeling a scalar (1-D) variable distribution, namely a first-order histogram of a typical imagedriven scalar variable (e.g., NDVI), as a combination of two or three MFs (e.g., corresponding to linguistic variables "low," "medium," and "high"). For example, Fig. 6 shows a typical histogram of a generic image variable, say, MIR1. This histogram is approximately bimodal and characterized by a right tail heavier than the left tail (positive skewness). Unfortunately, the histogram's valley detection algorithms proposed

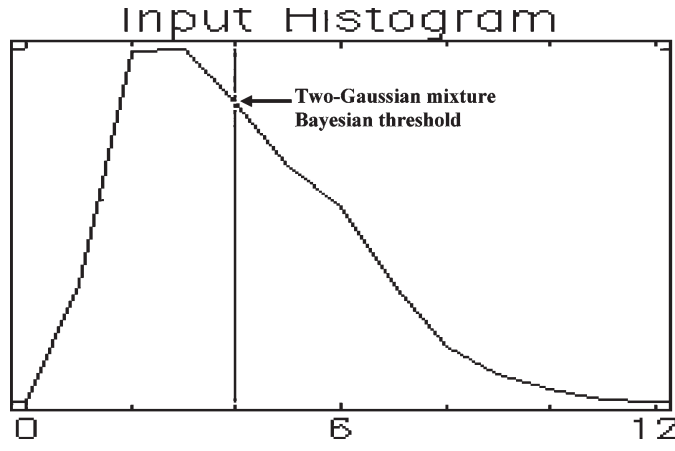

Fig. 7. Unimodal distribution properly partitioned into two qualitative high and low parts by the proposed histogram's valley detection technique.

in existing literature, e.g., in [2], are not robust to changes in a user-defined regularization parameter describing the width of irregular (local) peaks or valleys. To overcome this limitation, the automatic expectation-maximization (EM)-based two-Gaussian mixture density estimator (which is guaranteed to reach convergence) combined with a Bayesian thresholding criterion, which is taken from [18], is adopted to split into two a sample distribution assumed to be bimodal. It is noteworthy that even when the input histogram is unimodal, the proposed approach is capable of providing a correct solution (partition into two qualitative high and low parts) (see Fig. 7). In other words, the selected approach is robust against possible changes in the shape (number of modes, from one to two) of the input histogram.

\section{Cloud Cover Assessment}

In [38], a well-known automatic cloud cover assessment (ACCA) approach, which is suitable for dealing with the large cloud and land surface variability characterizing calibrated Landsat-7 ETM+ scenes, is proposed. The algorithm handles the cloud population in each scene uniquely by examining the image data twice. Pass one processing is designed to capture clouds and only clouds, i.e., errors of commission must be minimized, whereas errors of omission are accepted. Six different filters are used to isolate clouds and to eliminate cloudless areas and problem land surface features such as snow and sand. Three category classes result from pass one processing, namely, clouds, nonclouds, and an ambiguous group that is revisited in pass two, which is based on a thermal analysis of band TIR exclusively.

Filters adopted in pass one of ACCA, altogether with their adaptations exploited in this paper, are described below.

Filter 1: Band TM3, which is interpreted as brightness, is thresholded at 0.08. Pixels that exceed this threshold are passed to filter 2. In our approach, band TM3 is replaced by features Bright, Vis, NIR, MIR1, and MIR2, whose distinct thresholds have to be passed independently on a pixel basis.

Filter 2: NDSI, which is implemented as (13), is thresholded at 0.7. Pixels above this threshold are classified as snow (nonclouds), whereas pixels below this threshold are passed to filter 3. In our approach, (13) is replaced by (14), which is thresholded at 0.5 . 
TABLE III

Linguistic Labels Assigned to Fuzzy Sets Providing a Complete Partition of a Feature SPACE CONSISTING OF SCALAR VARIABLES BRIGHT, VIS, NIR, MIR1, MIR2, TIR, MIRTIR, NDSI, NDBBBI, NDVI, AND NDBSI DEFINED IN SECTION III-A

\begin{tabular}{|l|l|c|c|c|}
\hline \multicolumn{2}{|l|}{ Fuzzy sets } & \multicolumn{3}{|c|}{ Linguistic labels associated with (1-D) fuzzy sets } \\
\cline { 3 - 5 } \multicolumn{1}{|c|}{} & \multicolumn{1}{c|}{ Low } & Medium & High \\
\hline \multirow{4}{*}{$\begin{array}{l}\text { Input } \\
\text { features }\end{array}$} & Bright & LBright $(<40)$ & MBright & HBright $(>60)$ \\
\cline { 2 - 5 } & Vis & LVis $(<30)$ & MVis & HVis $(>50)$ \\
\cline { 2 - 5 } & NIR & LNIR $(<40)$ & MNIR & HNIR $(>60)$ \\
\cline { 2 - 5 } & MIR1 & LMIR1 $(<40)$ & MMIR1 & HMIR1 $(>60)$ \\
\cline { 2 - 5 } & MIR2 & LMIR2 $(<30)$ & MMIR2 & HMIR2 $(>50)$ \\
\cline { 2 - 5 } & TIR & LTIR $\left(<0.0^{\circ} \mathrm{C}\right)$ & MTIR & HTIR $\left(>28^{\circ} \mathrm{C}\right)$ \\
\cline { 2 - 5 } & MIRTIR & LMIRTIR $(<18000)$ & MMIRTIR & HMIRTIR $(>22000)$ \\
\cline { 2 - 5 } & NDSI & LNDSI $(<0.00)$ & MNDSI & HNDSI $(>0.50)$ \\
\cline { 2 - 5 } & NDBBBI & LNDBBBI $(<-0.20)$ & MNDBBBI & HNDBBBI $(>0.10)$ \\
\cline { 2 - 5 } & NDVI & LNDVI $(<0.36)$ & MNDVI & HNDVI $(>0.70)$ \\
\cline { 2 - 5 } & NDBSI & LNDBSI $(<-0.20)$ & MNDBSI & HNDBI $(>0.10)$ \\
\hline
\end{tabular}

Filter 3: Band TIR is thresholded at $300 \mathrm{~K}\left(27^{\circ} \mathrm{C}\right)$, which is a realistic cloud temperature maximum. All pixels with a temperature value below this threshold are passed to filter 4.

Filter 4: Band TM5/TM6 composite, which is implemented as the aforementioned feature MIRTIR [refer to (9)]. Sensitivity analysis demonstrated that a threshold setting of 225 works optimally. Pixel values above this threshold are labeled ambiguous in the cloud mask and are revisited in pass two. Pixels that fall below this threshold are passed to filter 5 .

Filter 5: Band TM4/TM3 ratio, which is known as RVI (refer to Section III-A2). RVI results in higher values for vegetation than for other scene features, including clouds. A threshold setting of 2.0 is used. Pixels that exceed this threshold are labeled ambiguous and are revisited in pass two. Pixels with ratios below this threshold are passed to filter 6 . In our testing cloud examples, this filter shows a large range of values, i.e., its discrimination utility appears minor. As a consequence, it is omitted in our approach.

Filter 6: Band TM4/TM5 ratio, which is inversely related to NDBSI [refer to (11)]. Rocks and sand tend to exhibit higher reflectance in band 5 than in band 4 , whereas the reverse is true for clouds. A threshold setting of 1.0 is reported to work effectively in eliminating highly reflective rocks and sands in desert landscapes. Pixels that fall below this threshold are labeled ambiguous and are revisited in pass two. Pixels with ratios that exceed this threshold are finally considered as belonging to clouds (either cold or warm). In our testing cloud cases, this filter shows a large range of values, i.e., its discriminating capability appears negligible. As a consequence, it is omitted in our approach.

In ACCA, pass two processing is engaged if and only if the following three conditions hold: 1) band TM4/TM5 ratio is greater than 0.5 (the image lacks highly illuminated rocks or sand); 2) colder cloud population exceeds $0.4 \%$ of the scene (pass two is not required if the scene is cloud-free); 3) mean temperature for the cloud class is less than $295 \mathrm{~K}$ (commission errors probably occurred if the mean temperature is this warm). In our approach, pass two is omitted.

\section{First-Level Processing of Kernel Spectral Categories- Step A: Feature Space Adaptive Grid Partitioning}

In line with the first-level processing of kernel spectral categories depicted in Fig. 4, Table III reports linguistic labels assigned to FSs (refer to Section II) whose rule patches (activation domains) provide an irregular but complete grid partition of an input (measurement) space consisting of features Bright, Vis, NIR, MIR1, MIR2, TIR, MIRTIR, NDVI, NDBSI, and NDSI (refer to Sections III-A1 and III-A2).

For the sake of simplicity, in the system implementation proposed below, FSs are rather implemented as crisp MFs (i.e., FSs employ a binary membership space; refer to Section II) whose activation domains are mutually exclusive and totally exhaustive. In particular, in each row in Table III, three FSs (whose fuzzy granules are, say, high, medium, and low) provide a complete nonoverlapping grid partition of a scalar variable.

Binary activation domains, where a scalar variable is partitioned into two sets, are adaptive to image statistics based on the automatic valley detection algorithm proposed in Section III-B.

Let us provide an example where (crisp) rule patches associated with linguistic variables low, medium, and high are automatically located through domain $[-1,1]$ of the scalar variable NDVI. Starting from the NDVI distribution, the valley detection algorithm presented in Section III-B selects an adaptive Bayesian threshold identified as NDVITH. The activation domain of the membership function associated with linguistic variable High is defined as the range (NDVITH, 1]. The activation domain of the membership function associated with linguistic variable Medium is defined as [NDVITL, NDVITH], where the (fuzzy) low NDVI threshold, identified as NDVITL, is heuristically estimated as NDVITL $=$ TOV $*$ $(\mathrm{NDVITH}-\min (\mathrm{NDVI}))+\min (\mathrm{NDVI})=[0.80 *(\mathrm{NDVITH}-$ $\min (\mathrm{NDVI}))+\min (\mathrm{NDVI})$, where tolerance value $\mathrm{TOV}=$ $0.80(80 \%)$. In other words, as a rule of thumb, a tolerance of $20 \%$ below the crisp NDVITH value is associated with linguistic variable Medium. For example, if NDVITH $\in[-1,1]$ is set to 0.70 by the valley detection algorithm, then $(0.80 *(\mathrm{NDVITH}-\min (\mathrm{NDVI})))+\min (\mathrm{NDVI})=(0.80 *$ $1.7-1)=0.36$. Thus, the activation domains of fuzzy sets LNDVI, MNDVI, and HNDVI become equal to $[-1,0.36)$, $[0.36,0.70]$, and $(0.70,1]$, respectively (see Table III). The activation domain of linguistic variable Low is defined as range $[-1$, NDVITL). Thus,

\section{if (NDVI $>$ NDVITH) then (HNDVI holds true)}

else if $(($ NDVI $\geq$ NDVITL $))$ and $($ NDVI $\leq$ NDVITH $))$ then (MNDVI holds true)

else (LNDVI holds true).

If NDBSIT $\in[-1,1]$ is set to 0.00 by the automatic valley detection algorithm, then $(0.85 *($ NDBSIT $-\min ($ NDBSI $)))+$ $\min ($ NDBSI $)=-0.15$. Thus, the NDBSI activation domains of FSs LNDBSI, MNDBSI, and HNDBSI become equal to $[-1,-0.15),[-0.15,0.00]$, and $(0.00,1]$, respectively (see Table III).

It is worthwhile to note that interesting image-invariant properties seem to emerge from our preliminary analysis of 
TABLE IV

LOGICAL EXPRESSIONS IMPLEMENTING FIRST-LEVEL KERNEL SPECTRAL RULES

\begin{tabular}{|c|c|c|c|}
\hline Index & Spectral rule name & $\begin{array}{l}\text { Spectral } \\
\text { rule } \\
\text { acronym }\end{array}$ & $\begin{array}{l}\text { Expression (where tolerance interval TV1 }=0.7>\text { TV2 }= \\
0.5 \text {; Input features: TM1-TM5, TM7: Landsat TM bands } \\
\text { 1-5 and 7) }\end{array}$ \\
\hline 1 & ThickCloudsSpectralRule & TKCL_SR & 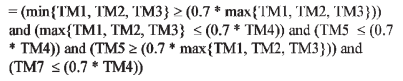 \\
\hline 2 & ThinCloudsSpectralRule & TNCL_SR & 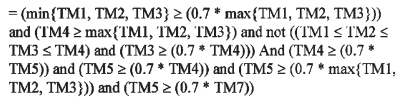 \\
\hline 3 & SnowOrIceSpectralRule & SNIC_SR & 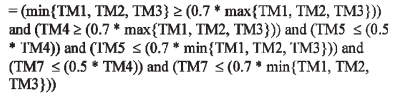 \\
\hline 4 & WaterOrShadowSpectralRule ${ }^{1}$ & WASH_SR & $\begin{array}{l}=(\mathrm{TM} 1 \geq \mathrm{TM} 2) \text { and }(\mathrm{TM} 2 \geq \mathrm{TM} 3) \text { and }(\mathrm{TM} 3 \geq \mathrm{TM} 4) \text { and } \\
\text { (TM4 } \geq \mathrm{TM} 5 \text { ) and (TM4 } 2 \text { TM7) }\end{array}$ \\
\hline 5 & PitbogOrGreenhouseSpectralRule & PBGH_SR & $\begin{array}{l}=(\mathrm{TM} 3 \geq(0.7 * \mathrm{TM} 1)) \text { and }(\mathrm{TM} 1 \geq(0.7 * \mathrm{TM} 3)) \text { and } \\
(\max \{\mathrm{TM} 1, \mathrm{TM} 2, \mathrm{TM} 3\} \leq(0.7 * \mathrm{TM} 4) \text { and }(\mathrm{TM} 5 \leq(0.7 * \\
\text { TM4)) and (TM3) } \geq(0.5 * \mathrm{TM} 5)) \text { and }(\min \{\mathrm{TM} 1, \mathrm{TM} 2, \mathrm{TM} 3\} \\
\left.\geq\left(0.7^{*} \mathrm{TM}\right)\right)\end{array}$ \\
\hline 6 & DominantBlueSpectralRule & DB_SR & $=\left(\mathrm{TM} 1 \geq\left(0.7^{*} \max \{\mathrm{TM} 2, \mathrm{TM} 3, \mathrm{TM} 4, \mathrm{TM} 5, \mathrm{TM} 7\}\right)\right)$ \\
\hline 7 & VegetationSpectralRule $^{2}$ & V_SR & $\begin{array}{l}=(\mathrm{TM} 2 \geq(0.5 * \mathrm{TM} 1)) \text { and }(\mathrm{TM} 2 \geq(0.7 * \mathrm{TM} 3)) \text { and }(\mathrm{TM} 3< \\
(0.7 * \mathrm{TM} 4)) \text { and }(\mathrm{TM} 4>\max \{\mathrm{TM} 1, \mathrm{TM} 2, \mathrm{TM} 3\}) \text { and }(\mathrm{TM} 5 \\
<(0.7 * \mathrm{TM} 4)) \text { and }\left(\mathrm{TM} 5 \geq\left(0.7^{*} \mathrm{TM} 3\right)\right) \text { and }(\mathrm{TM} 7<<0.7 * \\
\text { TM5)) }\end{array}$ \\
\hline 8 & RangelandSpectralRule $^{3}$ & R_SR & 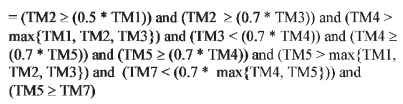 \\
\hline 9 & BarrenLandOrBuiltupOrCloudsSpectralRule ${ }^{4}$ & BBC_SR & 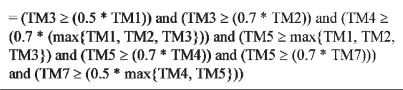 \\
\hline 10 & $\begin{array}{l}\text { FlatResponseBarrenLandOrBuiltUpSpectralR } \\
\text { ule }\end{array}$ & FBB_SR & $\begin{array}{l}=(\mathrm{TM} 5 \geq(0.7 * \max \{\mathrm{TM} 1, \mathrm{TM} 2, \mathrm{TM} 3, \mathrm{TM} 4, \mathrm{TM} 7\}))) \\
(\min \{\mathrm{TM} 1, \mathrm{TM} 2, \mathrm{TM} 3, \mathrm{TM} 4, \mathrm{TM} 7) \geq(0.5 * \mathrm{TM} 5)))\end{array}$ \\
\hline 11 & ShadowWithBarrenLandSpectralRule & SHB_SR & $\begin{array}{l}=(\mathrm{TM} 1 \geq \mathrm{TM} 2) \text { and }(\mathrm{TM} 2 \geq \mathrm{TM} 3) \text { and }(\mathrm{TM} 3 \geq(0.7 * \mathrm{TM} 4)) \\
\text { and (MM1 } \geq \mathrm{TM} 5) \text { and }(\mathrm{TM} 5 \geq(0.7 * \mathrm{TM} 4)) \text { and (TM5 } \geq(0.7 \\
\text { " TM7)) }\end{array}$ \\
\hline 12 & ShadowWithVegetationSpectralRule & SHV_SR & $\begin{array}{l}=(\mathrm{TM} 1 \geq \mathrm{TM} 2) \text { and }(\mathrm{TM} 2 \geq \mathrm{TM} 3) \text { and }(\mathrm{TM} 1 \geq(0.5 * \mathrm{TM} 4)) \\
\text { and }(\mathrm{TM} 3<(0.7 * \mathrm{TM} 4))) \text { and }(\mathrm{TM} 5<<0.7 * \mathrm{TM} 4)) \text { and }(\mathrm{TM} 3 \\
\left.\geq\left(0.7^{*} \mathrm{TM} 5\right)\right) \text { and }\left(\mathrm{TM} 7<\left(0.7^{*} \mathrm{TM} 4\right)\right)\end{array}$ \\
\hline 13 & ShadowCloudOrSnowspectralRule & $\begin{array}{l}\text { SHCLSN_ } \\
\text { SR }\end{array}$ & 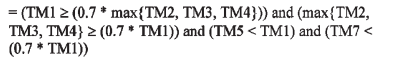 \\
\hline 14 & WetlandSpectralRule & WE_SR & $\begin{array}{l}=(\mathrm{TM} 1 \geq \mathrm{TM} 2) \text { and }(\mathrm{TM} 2 \geq \mathrm{TM} 3) \text { and }(\mathrm{TM} 1 \geq(0.7 * \mathrm{TM} 4)) \\
\text { and (TM3 }<\mathrm{TM} 4) \text { and (TM4 } \geq(0.7 * \mathrm{TM} 5) \text { ) and (TM5 } \geq(0.7 \\
\text { " TM4) ) and (TM3 } \geq(0.5 * \text { TMS)) and (TM5 } \geq \mathrm{TM} 7)\end{array}$ \\
\hline
\end{tabular}

${ }^{1}$ Exploitation of a tolerance threshold TV2 $=0.5$, which guarantees a tolerance value larger than TV1 $=0.7$, is also justified by the fact that very small reflectance values are involved with this spectral category. In this case, given two pixels featuring a low gray value, say, one and two, a gray level difference that equals one accounts for a relative change that equals $100 \%$ and $50 \%$, respectively.

Condition (TM2 $\geq(0.7 * \mathrm{TM} 3)$ ) is suitable for an image acquired during the leaf-on season.

R_SR shares conditions (TM2 $\geq(0.5 *$ TM1)), (TM2 $\geq(0.7 *$ TM3)) and (TM4 > max $\{$ TM1, TM2, TM3\}) with V_SR. Condition (TM2 $\geq(0.7 * T M 3))$ is suitable for an image acquired during the leaf-on season. $\mathrm{V} \_\mathrm{SR}$ and $\mathrm{R} \_\mathrm{SR}$ are disjunctive, the former rule requiring condition (TM5 $<(0.7 *$ TM4)), the latter requesting (TM5 $\geq(0.7 *$ TM4)). ${ }^{4} \mathrm{BBC}$ SR and V SR are disjunctive, the former rule requiring condition (TM5 $\left.\geq(0.7 * \mathrm{TM} 4)\right)$, the latter requesting (TM5 $<(0.7 * \mathrm{TM} 4))$.

a statistically meaningful sample set of distributions of the whole set of calibrated features Bright, Vis, NIR, MIR1, MIR2, TIR, MIRTIR, NDVI, NDBSI, and NDSI. These invariant properties are in line with the existing literature and/or are supported by physical/theoretical considerations. For example, condition "if (NDVI > 0.70) then HNDVI holds true" appears as a strong (necessary, but not sufficient) indication of the presence of the vegetated areas [36], [37]. Analogously, condition "if (NDBSI > 0.10) then HNDBSI holds true" appears as a strong (necessary, but not sufficient) indication of the presence of the barren land [9]. Once proved to be image independent, fixed thresholding of any input feature into two/three FSs (e.g., high, medium, and low) would increase the ease of use and decrease the computation time of the proposed automatic system of rules. To fulfill our objective of providing sufficient information for the implementation to be reproduced, the whole set of image-independent thresholds currently adopted in our crisp implementation of the proposed fuzzy rule-based system is reported in Table III.

\section{E. First-Level Processing of Kernel Spectral Categories- Step B: Nonhierarchical Computation of Kernel Spectral Rules}

In line with the first-level processing of the kernel spectral categories depicted in Fig. 4, Table IV reports the kernel spectral (fuzzy) rules (identified by suffix SR) currently implemented in the proposed system. As reported earlier in this text, kernel spectral rules are generated from well-known spectral signatures adopted as (fuzzy) templates in different portions of the electromagnetic spectrum (see Fig. 5). Each kernel spectral rule is implemented as a logical expression of scalar (numerical) variables combined with relational operators (e.g., $\geq$ ) and logical operators (e.g., logical AND). By definition, scalar variables are the Landsat bands TM1-TM5 and TM7 exclusively (i.e., excluding band TM6), which are calibrated into planetary reflectance values. In Table IV, two arbitrary spectral tolerance values, which are identified as TV1 $>$ TV2, are currently adopted to mimic fuzzy spectral rules. For example, if TV1 is set equal to 0.7 (refer to Section III-C), then TV2 may be set to 0.5 . Thus, given a generic numeric variable $X$, products $(\mathrm{TV} 1 * X)$ and $(\mathrm{TV} 2 * X)$ correspond to values $(X-30 \%$ of $X=70 \%$ of $X)$ and $(X-50 \%$ of $X=50 \%$ of $X)$, respectively.

Let us highlight the rationale in Table IV. As an example, the implemented vegetation spectral rule V_SR is compared with the prior spectral knowledge available in the form of the vegetation spectral signature shown in Fig. 5. In Table IV, V_SR is defined as

$$
\begin{aligned}
\mathrm{V} \_\mathrm{SR}= & (\mathrm{TM} 2 \geq(0.5 * \mathrm{TM} 1)) \text { and }(\mathrm{TM} 2 \geq(0.7 * \mathrm{TM} 3)) \\
& \text { and }(\mathrm{TM} 3<(0.7 * \mathrm{TM} 4)) \\
& \text { and }(\mathrm{TM} 4>\max \{\mathrm{TM} 1, \mathrm{TM} 2, \mathrm{TM} 3\}) \\
& \text { and }(\mathrm{TM} 5<(0.7 * \mathrm{TM} 4)) \text { and }(\mathrm{TM} 5 \geq(0.7 * \mathrm{TM} 3)) \\
& \text { and }(\mathrm{TM} 7<(0.7 * \mathrm{TM} 5)) .
\end{aligned}
$$

Exploiting the acronyms of spectral regions (namely, B, G, $\mathrm{R}$, NIR, MIR, and TIR) introduced in Table I, the lexical explanation of V_SR is stated as follows: V_SR is satisfied if ( $\mathrm{G}$ is not much "smaller" than $\mathrm{B}$ ) and $(\mathrm{G} \geq \mathrm{R}$, unless a "small" degree of tolerance) and ( $\mathrm{R}$ is "definitely smaller" than NIR) and (NIR > Visible) and (MIR1 is "definitely smaller" than NIR) and (MIR1 $\geq$ R, unless a "small" degree of tolerance) and (MIR2 is "definitely smaller" than MIR1). This fuzzy spectral rule is consistent with Fig. 5.

Let us also compare the vegetation spectral rule V_SR with the rangeland spectral rule $\mathrm{R} \_S R$, which is defined in Table IV as

$$
\begin{aligned}
\mathrm{R} \_\mathrm{SR}= & (\mathrm{TM} 2 \geq(0.5 * \mathrm{TM} 1)) \text { and }(\mathrm{TM} 2 \geq(0.7 * \mathrm{TM} 3)) \\
& \text { and }(\mathrm{TM} 3<(0.7 * \mathrm{TM} 4)) \\
& \text { and }(\mathrm{TM} 4>\max \{\mathrm{TM} 1, \mathrm{TM} 2, \mathrm{TM} 3\}) \\
& \text { and }(\mathrm{TM} 4 \geq(0.7 * \mathrm{TM} 5)) \text { and }(\mathrm{TM} 5 \geq(0.7 * \mathrm{TM} 4)) \\
& \text { and }(\mathrm{TM} 5>\max \{\mathrm{TM} 1, \mathrm{TM} 2, \mathrm{TM} 3\}) \\
& \text { and }(\mathrm{TM} 7<(0.7 * \max \{\mathrm{TM} 4, \mathrm{TM} 5\}) \\
& \text { and }(\mathrm{TM} 5 \geq \mathrm{TM} 7) .
\end{aligned}
$$


The lexical explanation of R_SR is stated as follows: R_SR is satisfied if ( $\mathrm{G}$ is not much "smaller" than $\mathrm{B})$ and $(\mathrm{G} \geq \mathrm{R}$, unless a "small" degree of tolerance) and ( $\mathrm{R}$ is "definitely smaller" than NIR) and (NIR > Visible) and (NIR and MIR1 are "similar") and (MIR1 > Visible) and (MIR2 is "definitely smaller" than the maximum between NIR and MIR1) and (MIR1 $\geq$ MIR2). It is worth noting that according to the implementation of fuzzy spectral rules R_SR and V_SR previously proposed, the intersection between R_SR and V_SR is always null, the former rule requiring (TM5 $\geq(0.7 * \mathrm{TM} 4))$, the latter $(\mathrm{TM} 5<(0.7 *$ TM4) $)$. Spectral rules BBC_SR and V_SR are disjunctive too, the former rule requiring condition (TM5 $\geq(0.7 *$ TM4 $))$, the latter asking for $(\mathrm{TM} 5<(0.7 *$ TM4)). Spectral rules SHV_SR and SHB_SR are disjunctive too (refer to Table IV).

\section{F. Second-Level Processing of Kernel Spectral Categories: Hierarchy of Logical Functions}

Unlike the flat first-level processing where kernel (fuzzy) spectral rules can be computed in parallel, the second-level processing of kernel spectral categories is a hierarchy of logical functions combining logical (Boolean) variables with logical operators (e.g., logical AND). These second-level input logical variables are the output of the kernel spectral rules and the FSs computed at first-level processing.

Table V provides a description of the implemented hierarchy of kernel spectral categories (i.e., types and strata), which are also called spectral candidate areas [2]. In its current implementation, the proposed system computes 67 kernel spectral categories, including category "unknown." Of the 67 spectral categories, 46 are leaves of the decision tree, identified by suffix LSC. The remaining kernel spectral categories, which are not leaves of the decision tree, are identified by suffix SC.

Intersections between the three FSs (i.e., high, medium, and low) detected along the two 1-D indexes NDVI and NDBSI (refer to Section III-D) generate nine rule patches provided with an intuitive symbolic meaning (as NDVI increases with vegetation, whereas NDBSI increases with built-up areas and barren land). As a function of these nine rule patches, Table VI shows the distribution across the decision space of the implemented kernel spectral categories described in detail in Table V.

Table VII provides the hierarchical set of logical expressions capable of generating the complete list of 67 kernel spectral categories described in Table V. This hierarchy of logical expressions is equivalent to an ordered sequence of the socalled hierarchical sublevels (HSLs), where each HSL consists of logical expressions eligible for being computed in parallel. To summarize, second-level kernel spectral categories are functions of first-level kernel spectral rules (refer to Table IV) and FSs defined in Table III, as well as second-level kernel spectral categories computed at lower HSLs. It is noteworthy that kernel spectral categories defined in Table VII are exhaustive and mutually exclusive.

\section{G. Stratified Classification/Clustering/Segmentation in Cascade to Spectral Rule-Based Preliminary Mapping}

It is well known that purely supervised (e.g., MLP-based) image classification approaches may be replaced by hybrid learning classification systems to better deal with the inherent spectral and spatial variability of RS imagery (see Sections I and II). Our aim is to investigate a cascade of a prior spectral knowledge-based preliminary mapping first stage with stratified (i.e., category-, class-, or application domain-specific) classification, clustering, or segmentation second-stage algorithms.

In general, the degree of complexity of a stratified classification/clustering/segmentation problem decreases monotonically with the symbolic meaning associated with informational strata. In other words, the degree of complexity of a stratified data analysis is expected to be (greatly) inferior to the analysis of the whole data set. An obvious advantage is that stratified data analysis may be conducted by simpler architectures (featuring fewer free parameters), which are easier to use and more capable of generalizing well [20]-[22].

Some comments on the proposed two-stage hybrid data learning approach, with special emphasis on stratified classification and segmentation, are provided below.

1) Stratified Multiple-Class Reference Sample Selection: Qualitative assessment (by an expert photointerpreter) of the quality of a preliminary map generated from the proposed spectral knowledge-based system should reveal that kernel spectral categories (i.e., types, layers, and strata) are consistent (in terms of one-to-one or many-to-one relationships) with land cover (informational) classes. For example, evergreen forest land (equivalent to USGS land cover 42) tends to be captured by spectral map categories AV_SC and SHV_LSC. In other words, kernel spectral layers AV_SC and SHV_LSC may be adopted as masks to ease detection and selection of reference samples belonging to, say, a land cover class identified as "evergreen forest." Additional reference samples belonging to other land covers of interest can be localized and selected at this stage by means of image masks (strata) generated by any combination of kernel spectral categories.

To summarize, reference samples selected by expert photointerpreters starting from first-stage kernel spectral strata may be employed to train second-stage supervised data learning classification algorithms (see Section I). In the framework of a bidirectional interaction between low- and high-level image processing (refer to Section I), detected land cover classes may be passed back on to the low-level processing stage to refine initial image mapping results, e.g., to reduce the extension of the EmptySU_LSC kernel spectral layer (refer to Table VII).

2) One-Class Classifiers and Outlier Detection: Let us consider a very common situation where a single target land cover falls in one (or more) kernel spectral category (categories) according to a one-category-to-one-class or many-categoriesto-one-class relationship. For example, land cover class "dark pine trees" tends to be captured by spectral information layer AV_SC (see also Table V). In this circumstance, a stratified one-class classifier should: 1) be trained by a reference data set extracted according to Section III-G-1 and 2) the one-class classifier should be run on a specific subset (stratum) of the image plane.

It is well known that any inductive learning classification requires as output, along with the classification index of a data input, a measure of confidence in this decision, or a refusal to make a decision if: 1) the data point lies in the overlapping region of two or more classes or 2 ) if it is found to come from a complete new class. The latter problem is known as novelty 
TABLE V

List OF 67 Kernel SPECTRAL CATEgories (TyPes, Strata, OR CANDidate AREas, Which ARE Identified by SuFFIX SC), INCLUDING 46 LEAVES OF THE DECISION TREE (IDENTIFIED BY SUFFIX LSC)
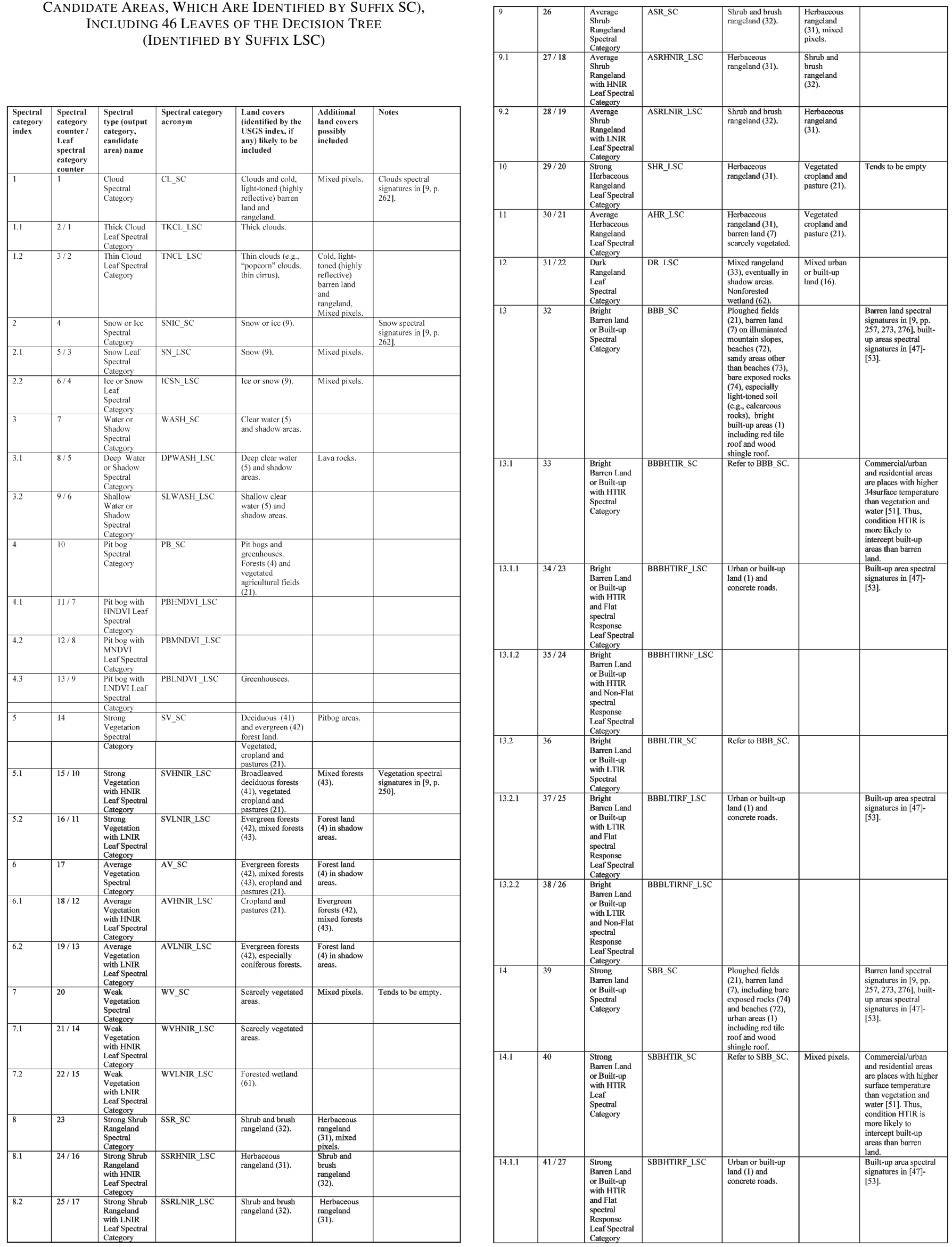
TABLE V

(Continued.) List of 67 Kernel SPectral Categories (Types, Strata, or CANDidate AREas, Which ARE IDENTIFIED B y SuFFIX SC), INCLUDING 46 LEAVES OF THE DECISION TREE (IDENTIFIED BY SUFFIX LSC)

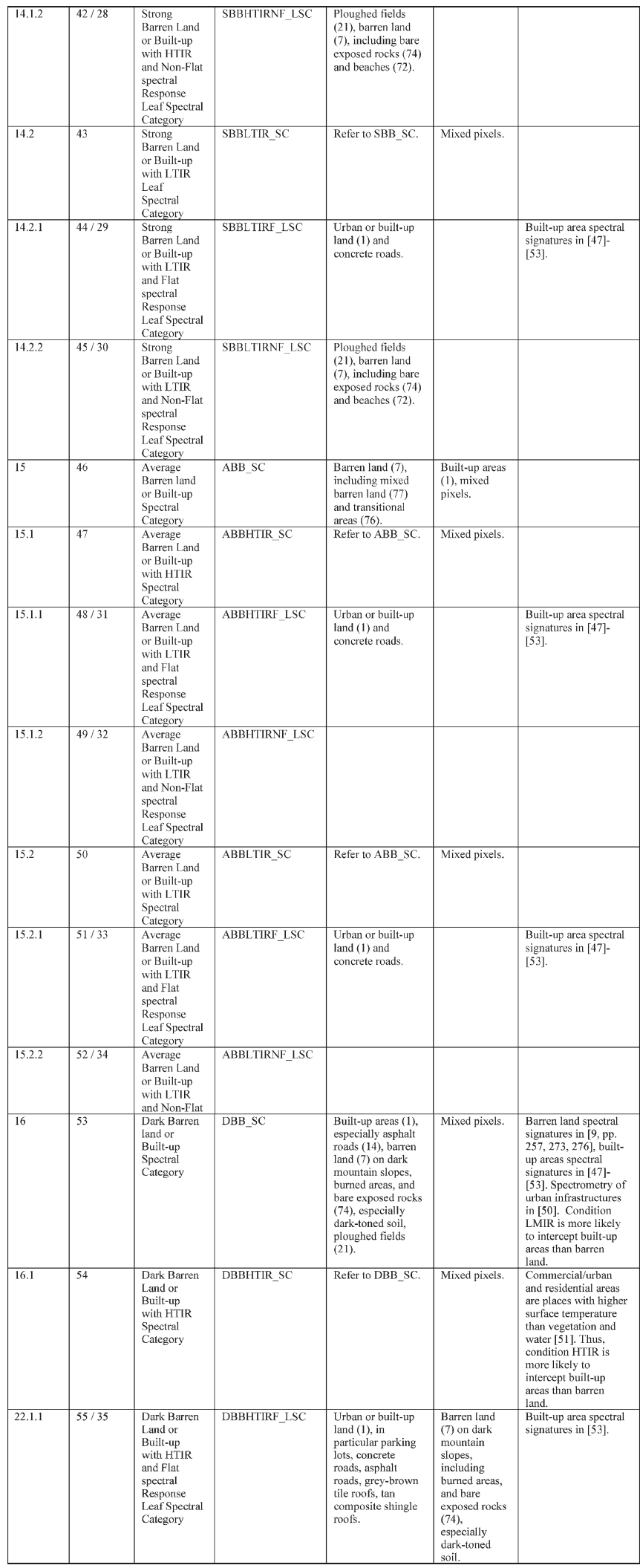

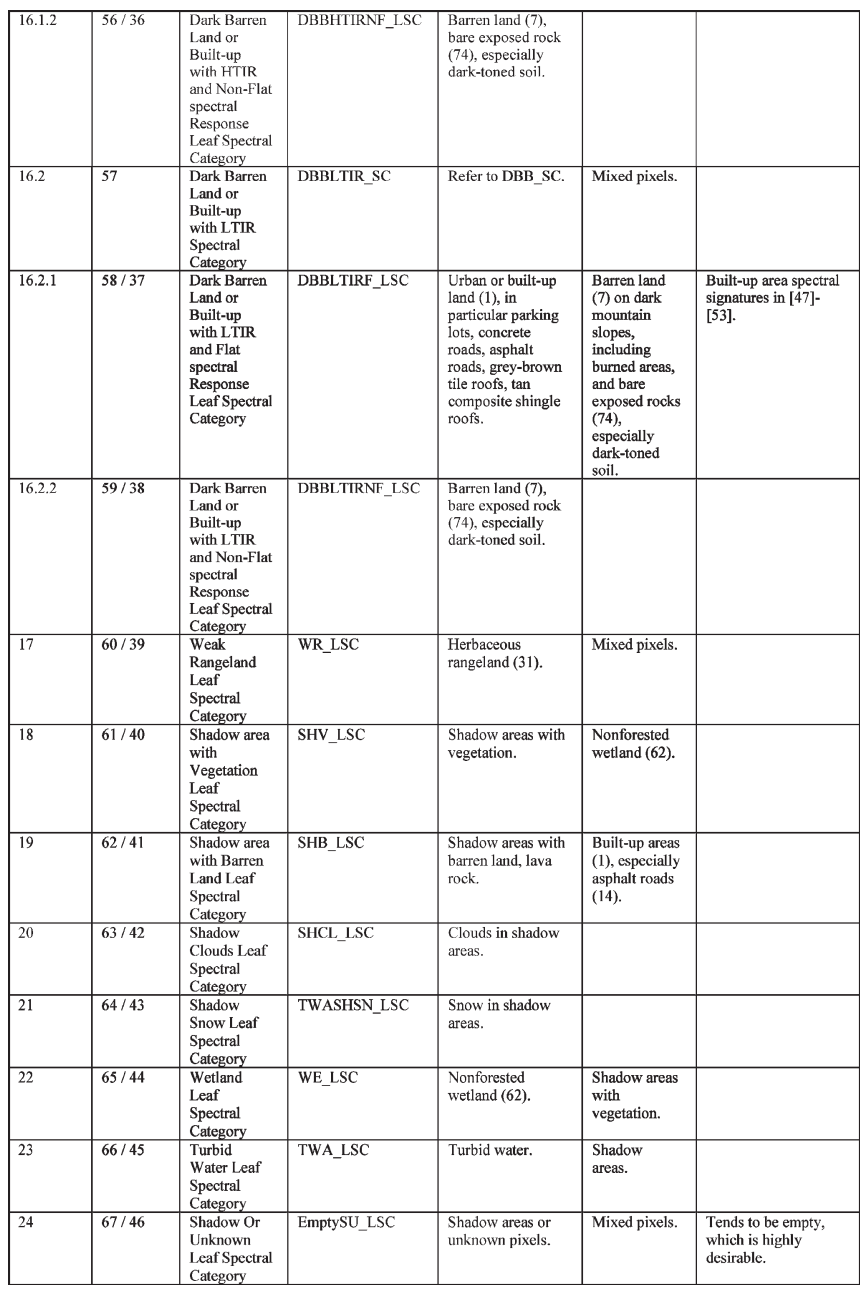

TABLE VI

Kernel Spectral Categories as a Combination of Fuzzy Sets High, Medium, and Low Detected Along Scalar Variables NDVI AND NDBSI. (DARK SHADING) RULE PATCHES MAINLY INVOLVED WITH RANGELAND. (UNSHADED) RULE PATCHES MAINLY INVOLVED WITH BARREN LAND AND BUILT-UP Areas. (Light Shading) Rule Patches Mainly INVOLVED WITH VEGETATION

\begin{tabular}{|c|c|c|c|}
\hline $\begin{array}{l}\text { Kernel spectral } \\
\text { categories }\end{array}$ & HNDBSI & MNDBSI & LNDBSI \\
\hline HNDVI & CL_SC, SHR_LSC & $\begin{array}{c}\text { CL_SC, SV_SC, SSR_SC, } \\
\text { SHCL_LSC, } \\
\text { TWASHSN_LSC }\end{array}$ & $\begin{array}{l}\text { CL_SC, SNIC_SC, } \\
\text { PB_SC, SV_SC, } \\
\text { SHCL_LSC, } \\
\text { TWASHSN_LSC }\end{array}$ \\
\hline MNDVI & $\begin{array}{l}\text { CL_SC, AHR_LSC, } \\
\text { SHV_LSC }\end{array}$ & $\begin{array}{c}\text { CL_SC, AV_SC, ASR SC, } \\
\text { SHV_LC, SHCL_LSC, } \\
\text { TWASHSN_SC, } \\
\text { WE_LSC }\end{array}$ & $\begin{array}{l}\text { CL_SC, SNIC SC, } \\
\text { PB_SC, AV_SC, } \\
\text { SHV_LSC, } \\
\text { SHCL_LSC, } \\
\text { TWASHSN_LSC, } \\
\text { WE LSC }\end{array}$ \\
\hline LNDVI & $\begin{array}{l}\text { CL_SC, WASH_SC, } \\
\text { DR_LSC, SHV_LSC, } \\
\text { SHB_LSC, BBB_SC, } \\
\text { SBB_SC, DBB_SC, } \\
\text { WR_LSC, TWA_LSC }\end{array}$ & $\begin{array}{c}\text { CL_SC, WASH_SC, } \\
\text { DR_LSC, SHV_LSC, } \\
\text { SHB_LSC, SHCL_LSC, } \\
\text { TWASHSN_LSC, } \\
\text { WE_LSC, BBB_SC, } \\
\text { ABB_SC, DBB_SC, } \\
\text { WR LSC, TWA_LSC }\end{array}$ & $\begin{array}{c}\text { CL_SC, SNIC_SC, } \\
\text { WASH_SC, PB_SC, } \\
\text { WV_SC, SHV_LSC, } \\
\text { SHB_LSC, } \\
\text { SHCL_LSC, } \\
\text { TWASHSN_LSC, } \\
\text { WE LSC, TWA LSC }\end{array}$ \\
\hline
\end{tabular}

detection, outlier detection, dissimilarity representation, or fault detection [54], [55]. If the target objects should be distinguished from the outlier objects, but only the information of the target class is assumed to be available although nothing is known about the outlier class, then the outlier detection problem is also known as one-class classification (OCC) [56], [57].

A typical approach to an OCC design in feature space is by describing the domain of the class in terms of typical input 
TABLE VII

Second-Level Processing of Kernel Spectral CATEgories. HIERARCHY OF LOGICAL EXPRESSIONS GENERATING A SET of Kernel SPeCtral Types That Are Totally EXHaUstive AND Mutually ExClusive

\begin{tabular}{|c|c|c|c|c|}
\hline Index & $\begin{array}{l}\text { Leaf } \\
\text { spectral } \\
\text { category } \\
\text { counter }\end{array}$ & $\begin{array}{l}\text { Spectral category } \\
\text { acronym }\end{array}$ & $\begin{array}{l}\text { Expression (where input features can be: spectral rules } \\
\text { described in Table IV, fuzzy sets described in Table III, } \\
\text { and lower hierarchical sub-levels), also refer to Fig. } 4\end{array}$ & $\begin{array}{l}\text { Second-level } \\
\text { spectral } \\
\text { category } \\
\text { computation- } \\
\text { Hierarchical } \\
\text { sub-level } \\
\text { (HSL) }\end{array}$ \\
\hline 1 & & CL_SC & $\begin{array}{l}\text { (TKCL_SR or TNCL_SR) and not (LBright or LVis or LNIR } \\
\text { or HNDSI or LMIR1 or LMIR2 or HIIR or HMIRTIR) }\end{array}$ & 1 \\
\hline 1.1 & 1 & TKCL_LSC & CL_SC and LMIRTIR & 2 \\
\hline 1.2 & 2 & TNCL_LSC & CL_SC and MMIRTIR & 2 \\
\hline 2 & & SNIC_SC & $\begin{array}{l}\text { SNIC_SR and LNDBSI and not(LBright or LVis or LNDSI or } \\
\text { LNIR or HMIR1 or HMIR2 or HTTR or CumulativeOR_SC') }\end{array}$ & 1 \\
\hline 2.1 & 3 & SN_LSC & SNIC_SC and HNDSI & 2 \\
\hline 2.2 & 4 & ICSN_LSC & SNIC_SC and MNDSI & 2 \\
\hline 3 & & WASH_SC & $\begin{array}{l}\text { WASH_SR and LBright and LVis and LNDVI and LNIR and } \\
\text { LMIR1 and LMIR2 and not (LTIR or CumulativeOR_SC) }\end{array}$ & 1 \\
\hline 3.1 & 5 & DPWASH_LSC & WASH_SC and HNDSI & 2 \\
\hline 3.2 & 6 & SLWASH_LSC & WASH_SC and not(HNDSI) & 2 \\
\hline 4 & & PB_SC & $\begin{array}{l}\text { PBGH_SR and LMIR1 and LMIR2 and LNDBSI and not } \\
\text { (LNIR or CumulativeOR_SC) }\end{array}$ & 1 \\
\hline 4.1 & 7 & PBHNDVI_LSC & PB_SC and HNDVI & 2 \\
\hline 4.2 & 8 & PB MNDVI_LSC & PB_SC and MNDVI & 2 \\
\hline 4.3 & 9 & PB LNDVI_LSC & PB_SC and LNDV1 & 2 \\
\hline 5 & & SV_SC & $\begin{array}{l}\text { V_SR and HNDVI and not(HMIR1 or HMIR2 or HNDBSI or } \\
\text { CumulativeOR_SC) }\end{array}$ & 2 \\
\hline 5.1 & 10 & SVHNIR_LSC & SV_SC and HNIR & 3 \\
\hline 5.2 & 11 & SVLNIR_LSC & SV_SC and not(HNIR) & 3 \\
\hline 6 & & AV_SC & $\begin{array}{l}\text { (V_SR or SHV_SR) and MNDVI and not (HMIR1 or HMIR2 } \\
\text { or HNDBSI or CumulativeOR_SC or DB_SR) }\end{array}$ & 2 \\
\hline 6.1 & 12 & AVHNIR_LSC & AV_SC and HNIR & 3 \\
\hline 6.2 & 13 & AVLNIR_LSC & $\mathrm{AV} \_\mathrm{SC}$ and not(HNIR) & 3 \\
\hline 7 & & WV_SC & $\begin{array}{l}\text { (V_SR or R_SR or SHV_SR) and LNDVI and LNDBSI and } \\
\text { LMR1 and LMR2 and not (CumulativeOR_SC or DB_SR) }\end{array}$ & 2 \\
\hline 7.1 & 14 & WVHNIR_LSC & WV_SC and HNIR & 3 \\
\hline 7.2 & 15 & WVLNIR_LSC & WV_SC and not(HNIR) & 4 \\
\hline 8 & & SSR_SC & $\begin{array}{l}\text { R_SR and HNDVI and MNDBSI and not } \\
\text { (CumulativeOR__C) }\end{array}$ & 3 \\
\hline 8.1 & 16 & SSRHNIR_LSC & SSR_SC and HNIR & 4 \\
\hline 8.2 & 17 & SSRLNIR_LSC & SSR_SC and not(HNIR) & 4 \\
\hline 9 & & ASR_SC & $\begin{array}{l}\text { R_SR and MNDVI and MNDBSI and not (CumulativeOR_SC } \\
\text { or SHV_SR or WE_SR) }\end{array}$ & 3 \\
\hline 9.1 & 18 & ASRHNIR_LSC & ASR_SC and HNIR & 4 \\
\hline 9.2 & 19 & ASRLNIR_LSC & ASR_SC and not(HNIR) & 4 \\
\hline 10 & 20 & SHR_LSC & R_SR and HNDVI and HNDBSI and not (CumulativeOR_SC) & 2 \\
\hline 11 & 21 & AHR_LSC & (R_SR or BBC_SR) and MNDVI and HNDBSI and not & 2 \\
\hline 12 & 22 & DR_LSC & $\begin{array}{l}\text { (V_SR or R_SR) and LNDVI and LMIR } 2 \text { and not (IINIR or } \\
\text { HMIRI or LNDBSI or CumulativeOR_SC) }\end{array}$ & 2 \\
\hline 13 & & BBB_SC & $\begin{array}{l}\text { BBC_SR and HNIR and HMIR2 and LNDVI and not } \\
\text { (LNDBSI or LMIR1 or CumulativeOR_SC) }\end{array}$ & 2 \\
\hline 13.1 & & BBBHTTR_SC & BBB_SC and HTIR & 3 \\
\hline 13.1.1 & 23 & BBBHTIRF_LSC & BBBHTIR_SC and not (LNDBBBI) & 4 \\
\hline 13.1.2 & 24 & BBBHTIRNF_LSC & BBBHTIR_SC and not (BBBHTIRF_LSC) & 5 \\
\hline 13.2 & & BBBLTIR_SC & BBB_SC and not(HTIR) & 3 \\
\hline 13.2.1 & 25 & BBBLTIRF_LSC & BBBLTIR_SC and not (LNDBBBI) & 4 \\
\hline 13.2 .2 & 26 & BBBLTIRNF_LSC & BBBLTIR_SC and not (BBBLTIRF_LSC) & 5 \\
\hline 14 & & SBB_SC & $\begin{array}{l}\text { (BBC_SR or FBB_SR) and LNDVI and HNDBSI and } \\
\text { not(HNIR or LMIR1 or CumulativeOR_SC) }\end{array}$ & 2 \\
\hline 14.1 & & SBBHTTR_SC & SBB_SC and HTIR & 3 \\
\hline 14.1.1 & 27 & SBBHTTRF_LSC & SBBHTIR_SC and (DB_SR or FBB_SR) & 4 \\
\hline 14.1.2 & 28 & SBBHTIRNF_LSC & SBBHTIR_SC and not (SBBHTIRF_LSC) & 5 \\
\hline 14.2 & & SBBLTIR_SC & SBB_SC and not(HTIR) & 3 \\
\hline 14.2.1 & 29 & SBBLTIRF_LSC & SBBLTIR_SC and (DB_SR or FBB_SR) & 4 \\
\hline 14.2 .2 & 30 & SBBLTIRNF_LSC & SBBLTIR_SC and not (SBBLTTRNF_LSC) & 5 \\
\hline 15 & & ABB_SC & $\begin{array}{l}\text { (BBC_SR or FBB_SR) and LNDVI and MNDBSI and not } \\
\text { (HNIR or LMIR1 or CumulativeOR_SC) }\end{array}$ & 2 \\
\hline 15.1 & & ABBHTIR_SC & ABB_SC and HTIR & 3 \\
\hline 15.1.1 & 31 & ABBHTIRF_LSC & ABBHTIR_SC and not (LNDBBBI) & 4 \\
\hline 15.1 .2 & 32 & ABBHTIRNF_LSC & ABBHTIR_SC and not (ABBHTTRF_LSC) & 5 \\
\hline 15.2 & & ABBLTIR_SC & ABB_SC and not(HTIR) & 3 \\
\hline 15.2 .1 & 33 & ABBLTIRF_LSC & ABBLTIR_SC and not (LNDBBBI) & 4 \\
\hline 15.2 .2 & 34 & ABBLTIRNF_LSC & ABBLTIR_SC and not (ABBLTIRF_LSC) & 5 \\
\hline 16 & & DBB_SC & $\begin{array}{l}\text { (BBC_SR or FBB_SR) and LNDVI and LMIR1 and } \\
\text { not(HNIR or HMIR2 } 2 \text { or LNDBSI or CumulativeOR_SC) }\end{array}$ & 3 \\
\hline 16.1 & & DBBHTIR_SC & DBB_SC and HTIR & 4 \\
\hline 16.1.1 & 35 & DBBHTIRF_LSC & DBBHTIR_SC and (DB_SR or FBB_SR) & 5 \\
\hline 16.1 .2 & 36 & DBBHTIRNF_LSC & DBBHTIR_SC and not (DBBHTIRF_LSC) & 6 \\
\hline 16.2 & & DBBLTIR_SC & DBB_SC and not(HTIR) & 4 \\
\hline 16.2 .1 & 37 & DBBLTTRF_LSC & DBBLTIR_SC and (DB_SR or FBB_SR) & 5 \\
\hline 16.2 .2 & 38 & DBBLTIRNF_LSC & DBBLTIR_SC and not (DBBLTIRF_LSC) & 6 \\
\hline 17 & 39 & WR_LSC & R_SR and LNDVI and not(LNDBSI or CumulativeOR_SC) & 4 \\
\hline 18 & 40 & SHV_LSC & $\begin{array}{l}\text { DB_SR and SHV_SR and LBright and LVis and LNIR and } \\
\text { LMIR1 and LMIR2 and not (HNDVI or CumulativeOR_SC) }\end{array}$ & 5 \\
\hline 19 & 41 & SHB_LSC & $\begin{array}{l}\text { DB_SR and SHB_SR and LBright and LVis and LNDVI and } \\
\text { LNIR and LMIR1 and LMIR2 and not (CumulativeOR_SC) }\end{array}$ & 6 \\
\hline 20 & 42 & SHCL_LSC & $\begin{array}{l}\text { DB_SR and SHCLSN_SR and not (HNDSI or LNIR or } \\
\text { LBright or LVis or HNDBSI or HTIR or CumulativeOR_SC) }\end{array}$ & 7 \\
\hline
\end{tabular}

\begin{tabular}{|l|c|l|l|c|}
\hline 21 & 43 & TWASHSN_LSC & $\begin{array}{l}\text { DB_SR and SHCLSN_SR and HNDS1 and LNIR and LMIR1 } \\
\text { andLMMR2 and not(HBright or HVis or HNDBSI or HTTR or } \\
\text { CumulativeOR_SC) }\end{array}$ & 7 \\
\hline 22 & 44 & WE_LSC & $\begin{array}{l}\text { DB_SR and WE_SR and LBright and LVis and LNIR and } \\
\text { LMIR1 and LMIR2 and not (HNDVI or HNDBSI or LNDSI } \\
\text { or CumulativeOR_SC) }\end{array}$ & $\mathbf{8}$ \\
\hline 23 & 45 & TWA_LSC & $\begin{array}{l}\text { DB_SR and LNDVI and LMIR1 and LMIR2 and not (HBright } \\
\text { or HVis or HNIR or LNDSI or CumulativeOR_SC) }\end{array}$ & 9 \\
\hline 24 & 46 & EmptySU_LSC & 1 and not (CumulativeOR_SC) & 10 \\
\hline
\end{tabular}

samples, i.e., an ideal OCC designed in feature space should not respond to data points that lie far away from the boundary of its training data set. This inductive learning principle is also known as strict generalization [58]. In strict generalization, a traditional probability density function (pdf) estimation of the training data would allow us to consider as outliers all the testing samples that fall in input regions with low density. Unfortunately, due to the curse of dimensionality, traditional pdf estimation requires a number of samples (growing exponentially with input space dimensionality $N$ ) that soon becomes prohibitive (as $N$ becomes greater than 10, e.g., in hyperspectral data) to avoid the majority of the histogram bins being empty [15]. Thus, an efficient OCC tries to determine in feature space a minimal volume hypersphere around the reference data based on a small (reduced) number of selected prototype objects [56].

Traditionally, a good OCC should have a large fraction of true positive (TP), which is proportional to the so-called sensitivity, and a small fraction of false positive (FP), which is tantamount to saying a large fraction of true negative (TN), which is proportional to the so-called specificity, where $\mathrm{TN}+$ $\mathrm{FP}=$ total negative samples. The dependency of TP as a function of FP is typically shown in a so-called receiver operating characteristic (ROC) curve where: 1 ) any increase in sensitivity causes a decrease in specificity and 2) the closer the curve follows the left-hand border and then the top border of the ROC space, the more accurate the test is [59].

3) Stratified Segmentation Versus Category-Oriented Knowledge-Driven Segmentation: In the image-processing literature, image segmentation techniques are divided into: 1) general-purpose segmentation approaches and 2) categoryoriented knowledge-driven algorithms [1]. In general-purpose segmentation techniques, there is a loose or no relationship between the adopted segmentation criteria and domain-specific prior knowledge about target classes. In this case, if the quality of the segmentation map is poor, it may be difficult for a user to recover from segmentation errors. As a consequence, general-purpose segmentation approaches are not very useful in practice.

To avoid this limitation, i.e., to obtain more robust and reliable segmentation solutions, category-oriented segmentation algorithms are developed. In this case, spectral and spatial prior knowledge-based constraints concerning a target land cover (e.g., roads [60]) are integrated into the segmentation criteria (constraints).

It is noteworthy that a two-stage hybrid data learning framework consisting of a cascade between a kernel spectral category detection first stage and a stratified image segmentation second stage [which was proposed as a potential application domain of the novel spectral rule-based system (refer to Section I, point 1.c.ii)] is similar, but not equivalent, to a highly recommended single-stage knowledge-driven segmentation approach. In particular, the former two-stage cascade approach does not require integration of any spectral or spatial domain-specific 
TABLE VIII

Main Characteristics of SOME Rule-Based Classifiers Found IN THE RS LITER ATURE COMPARED TO THE PROPOSED SPECTRAL KNOWLEDGE-BASED SYSTEM

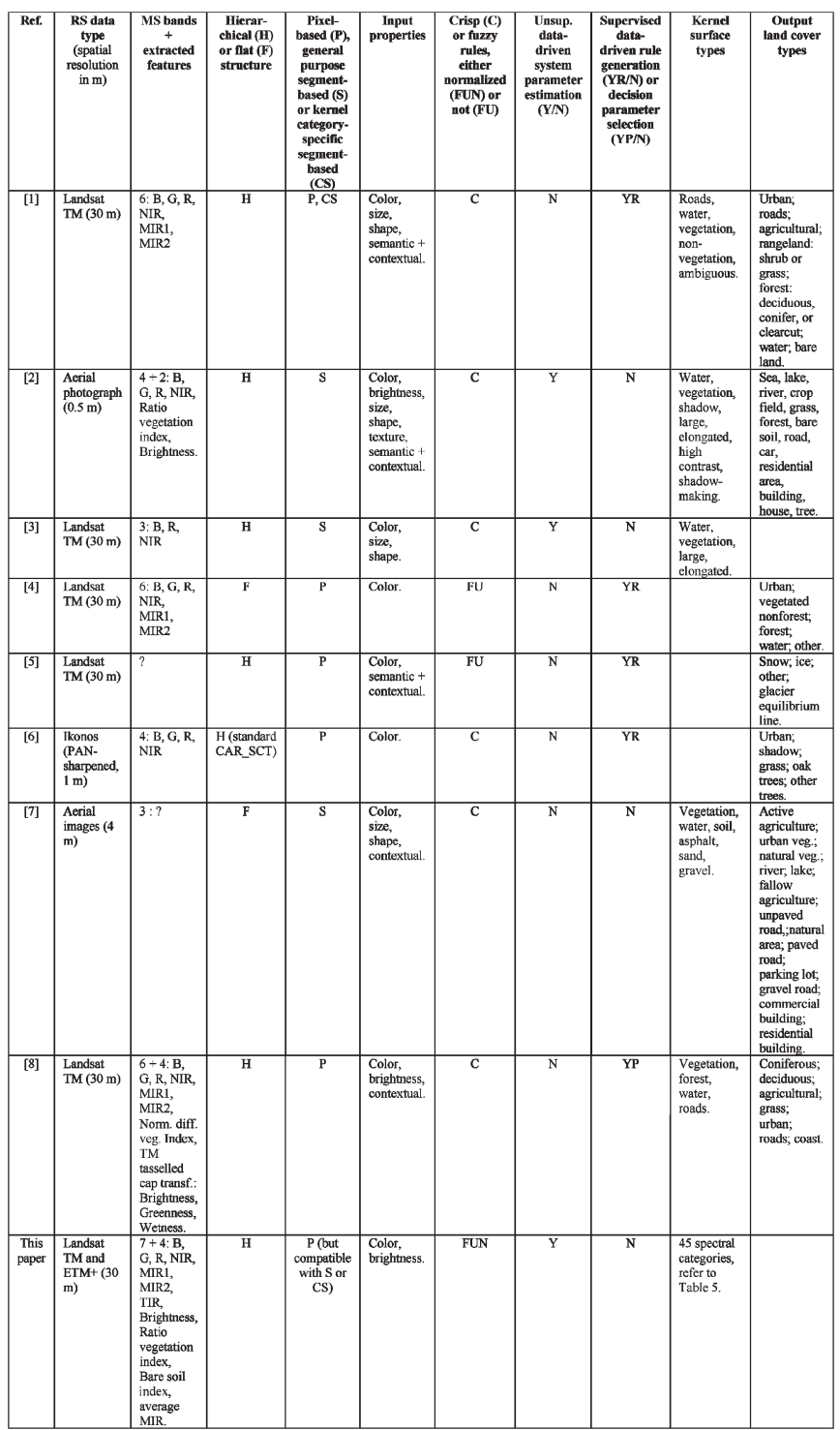

knowledge about a target land cover (e.g., roads) into the image segmentation algorithm (as in [60], for example). In other words, rather than employing the entire RS image as input, a general-purpose segmentation algorithm should be run separately on different image strata where the segmentation problem has been made easier to solve by exploiting spectral prior knowledge.

\section{Degree of Novelty of the Proposed RULE-BASED SYSTEM}

To assess its potential degree of novelty, the spectral knowledge-based fuzzy classifier proposed in Section III is compared against several rule-based classifiers published in the RS literature in recent years. In particular, Table VIII compares alternative rule-based systems based on their relevant design properties. Some of these salient functional properties are discussed below.

\section{A. Pixel-Versus Object-Based Systems of Rules}

It is worth noting that unlike the purely spectral contextinsensitive nature of the proposed system of rules (refer to Section I), well-known fuzzy decision rule-based classifiers, either found in the RS literature (e.g., [1] and [2]) or implemented in commercial image-classification software toolboxes in recent years (e.g., [34], whose advent has actually fostered new interest in RS image segmentation methods), apply decision rules to homogeneous image object primitives rather than pixels. Both rule-based approaches, whether pixelor object-based, have intrinsic advantages and limitations, which are summarized below.

A well-known drawback of purely supervised contextinsensitive classification approaches is that they are often unable to account for the large spectral and structural heterogeneity (clutter) of RS imagery (in particular, VHR RS imagery; refer to Section I, point 1.c.iii). As a consequence, their thematic maps can be affected by the so-called salt-andpepper classification noise effects, i.e., these thematic maps tend to lack spatial coherence.

To circumvent this problem, a common RS practice is to detect homogeneous image object primitives (segments) prior to classification (e.g., see [1], [2], and [34]). Unfortunately, image segmentation, like any low-level signalprocessing task, is inherently ill-posed, i.e., there is no single goal for image segmentation/edge detection algorithms as is the case of data clustering. Although well known in existing literature (e.g., refer to [22]-[26]), the problem of segmentation ill-posedness is rather ignored in RS common practice. Beside its inherent ill-posedness, additional potential limitations of an image segmentation stage prior to classification are a heavy computational load, large memory occupation, and complex maintenance of a segment property table [2].

Due to its capability of effectively combining multiple sources of fuzzy spectral evidence, the proposed pixel-based system of rules is not affected by the salt-and-pepper classification noise effects, i.e., it tends to generate no artifact while preserving spatial coherence (see Section V). This implies that the proposed image classifier requires no input image segmentation preprocessing stage, which is equivalent to an intrinsically ill-posed edge-preserving low-pass image filter. To summarize, the proposed rule-based classification approach is expected to preserve genuine but small image details while reducing computation time and the number of arbitrary userdefined free parameters required by the alternative segmentbased fuzzy classifiers (e.g., [34]).

\section{B. Class of Rule-Based Systems Detecting Kernel Image Information Layers}

Table VIII reveals that the proposed approach belongs to the same class of systems presented in [1]-[3]. In particular, the proposed system appears to be capable of replacing/augmenting the kernel information extraction modules designed for Landsat TM imagery and presented in [1] and [3]. Despite being developed for satellite data rather than aerial imagery, the proposed rule-based classifier is also suitable for providing new spectral rules to the kernel region extraction stage designed for aerial 
image interpretation in [2]. To summarize, our paper addresses the problem of kernel spectral surface type detection in a way that appears to be new and of interest to the RS readership.

\section{EXPERIMENTAL RESUlTS}

The efficacy of the proposed spectral rule-based system, whether employed as standalone or in cascade with a stratified classification (refer to points 1.a and 1.c in Section I), and its robustness to changes in the input data set are tested in two realistic RS image classification problems where Landsat-5 TM and 7 ETM+ imagery depicting extended surface areas are provided with little or no ground truth knowledge.

It is worthwhile to recall that any classification overall accuracy (OA) (precision) probability estimate $p_{\mathrm{OA}} \in[0,1]$ is a random variable (sample statistic) with a confidence interval (error tolerance) associated with it, i.e., it is a function of the specific training and testing sets being used [22]. Based on the assumption that reference samples are independent and identically distributed (which does not strictly hold in our case due to spatial autocorrelation between neighboring pixels), confidence interval (error tolerance) $\delta$ associated with probability estimate $p_{\mathrm{OA}}$ is computed according to [22]

$$
\delta=\sqrt{\frac{(1.96)^{2} \cdot p_{\mathrm{OA}} \cdot\left(1-p_{\mathrm{OA}}\right)}{R F}}
$$

where $R F$ is the cardinality of the reference data set at hand. For each $i$ th class simultaneously involved in the classification process, with $i=1, \ldots, L$, where $L$ is the total number of classes, it is possible to prove that (refer to [45, p. 294])

$$
\delta_{i}=\sqrt{\frac{\chi_{(1,1-\alpha / L)}^{2} \cdot p_{\mathrm{OA}, i} \cdot\left(1-p_{\mathrm{OA}, i}\right)}{R F_{i}}}, \quad i=1, \ldots, L
$$

where $\alpha$ is the desired level of significance, i.e., the risk that the actual error is larger than $\delta_{i}$ (e.g., $\alpha=0.01$ ), $1-\alpha$ is the level of confidence (e.g., 0.99), and $\chi_{1,1-\alpha / L}^{2}$ is the upper (1$\alpha) / L * 100$ th percentile of the chi-square distribution with one degree of freedom. For example, if $L$ equals 4 or 5 , then the upper $(0.99) / L * 100$ th percentile of the chi-square distribution is $\chi_{(1,0.25)}^{2}=0.102$.

\section{A. Landsat-7 ETM+}

By means of RS imagery and geospatial metrics combined with other available geo information, forest/nonforest binary classification maps are planned to be generated from Landsat5 TM scenes acquired in the late 1980s and Landsat-7 ETM+ scenes acquired around the year $2000 .^{3}$ In this binary classification framework, the proposed prior spectral knowledgebased system of rules is tested to provide a preliminary binary partition of the input Landsat imagery into vegetation and nonvegetation surface types, which is in line with the first hierarchical level of the land cover classification scheme shown in Fig. 2. This initial classification binary partition would allow

\footnotetext{
${ }^{3}$ The European Union (EU) Joint Research Centre-INFOREST biodiversity action aims at assessing forest types and their spatial changes over several decades at a pan-European scale [42], [43].
}

further detection of forest areas within the vegetated image layer exclusively (i.e., by ignoring nonvegetated image areas).

In our experiment, first, an orthorectified Landsat scene is extracted from the Global Land Cover Facility (GLCF) Landsat ETM+ data set [61]. The selected scene (path/raw: 191/30, acquired on August 3, 2001), which is $6999 \times 8065$ pixels in size, depicts an area located in central Italy (Marche region). Although the ETM+ MS bands (1-5 and 7) are radiometrically calibrated into planetary reflectance, the thermal band ETM+ 62 is calibrated into at-satellite temperature of the viewed Earth-atmosphere system based on equations reported on the Landsat-7 Handbook [10], whose calibration parameters are extracted from the scene's metafile. The image calibration result is shown in Fig. 8, which depicts a false color composition (i.e., R: TM5, G: TM4, and B: TM1) of the selected Landsat-7 ETM+ image. In Fig. 8, vegetated areas appear bright green (including forest areas and crop fields), water bodies appear dark and light blue, whereas nonvegetated land (including ploughed agricultural fields, urban areas, and barren land) looks pink-lilac. Finally, the spectral rule-based map, which is shown in Fig. 9, is generated from the orthorectified calibrated Landsat scene shown in Fig. 8. In Fig. 9, spectral types of interest are depicted in pseudocolors according to the following legend: vegetated candidate areas (namely, SV_SC, AV_SC, WV_SC, SSR_SC, ASR_SC, SHR_LSC, AHR_LSC, DR_LSC, SHV_LSC, WE_LSC, and WR_LSC) are in different shades of green (from bright to dark), water bodies are dark (WASH_SC) and light blue (TWA_LSC, TWASHSN_SNC), whereas barren land and built-up candidate areas (namely, BBB_SC, SBB_SC, ABB_SC, DBB_SC, and SHB_LSC; see below for more details) are in brown and gray shades.

1) Qualitative Map Assessment: For the sake of simplicity, let us focus our visual analysis on a small subset of Figs. 8 and 9, which are shown in Figs. 10 and 11, respectively, which are $400 \times 300$ pixels in size. In Fig. 10, forest areas appear in different shades of (false color) green due to the changing illumination conditions and the nonstationary surface type properties. In Fig. 11, most forest areas are classified as SV_SC (bright green), with the exception of forests located on the steepest shadow slopes or along the transitional boundaries between forest and nonforest areas (where mixed pixels occur), which are assigned to spectral types AV_SC (dark green) and SHV_LSC (very dark green). In line with the theoretical expectations, the vegetation detection system seems to be rather insensitive to shadow areas.

Figs. 12 and Fig. 13 show a larger subset of Figs. 8 and Fig. 9, respectively, which are $1200 \times 1200$ pixels in size. It is worthwhile to note that the pixels of Fig. 12, belonging to land cover classes whose within-class spectral variability is large, such as urban areas and agricultural land, are mapped into kernel spectral categories whose spatial coherence remains high in Fig. 13. This appears as a relevant advantage of the proposed per-pixel prior knowledge-based classifier over the traditional pixel-based inductive learning mapping systems that are typically affected by the salt-and-pepper classification noise effects (see Section VI-A).

2) Quantitative Map Assessment: Starting from the output map consisting of kernel spectral types shown in Fig. 9, a vegetation/nonvegetation binary map is obtained by aggregating spectral types as described in Table IX. According to this binary 


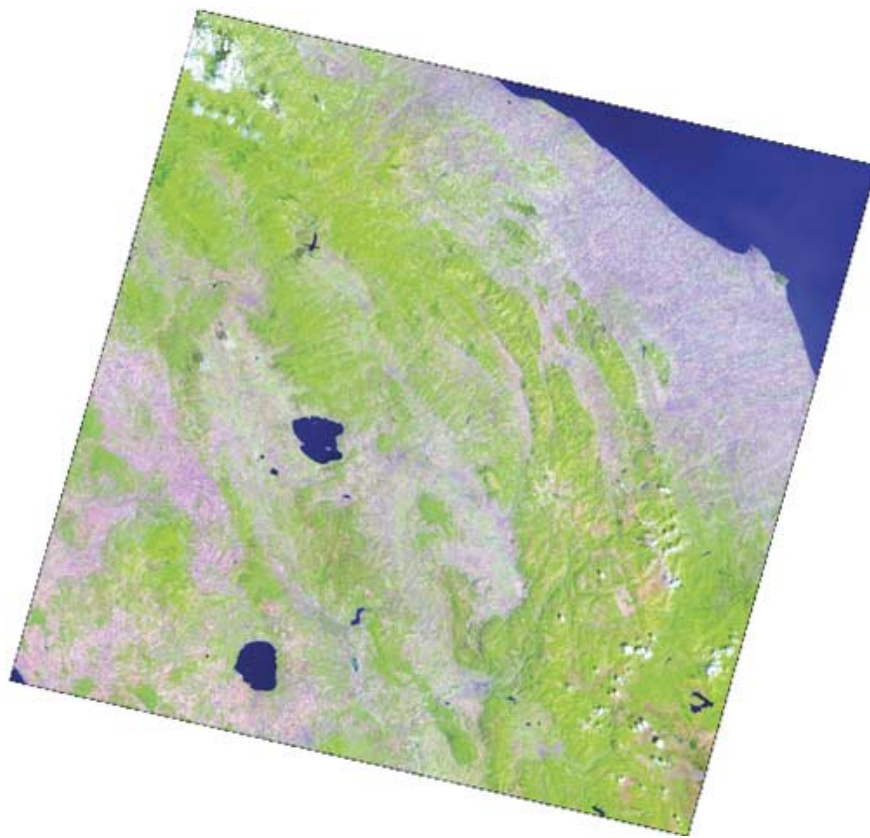

Fig. 8. Orthorectified and calibrated Landsat-7 ETM+ image (in false colors, $\mathrm{RGB}=$ bands 541 ) of the test area located in central Italy. Acquisition date: August 3, 2001. Image size: $6999 \times 8065$ pixels.

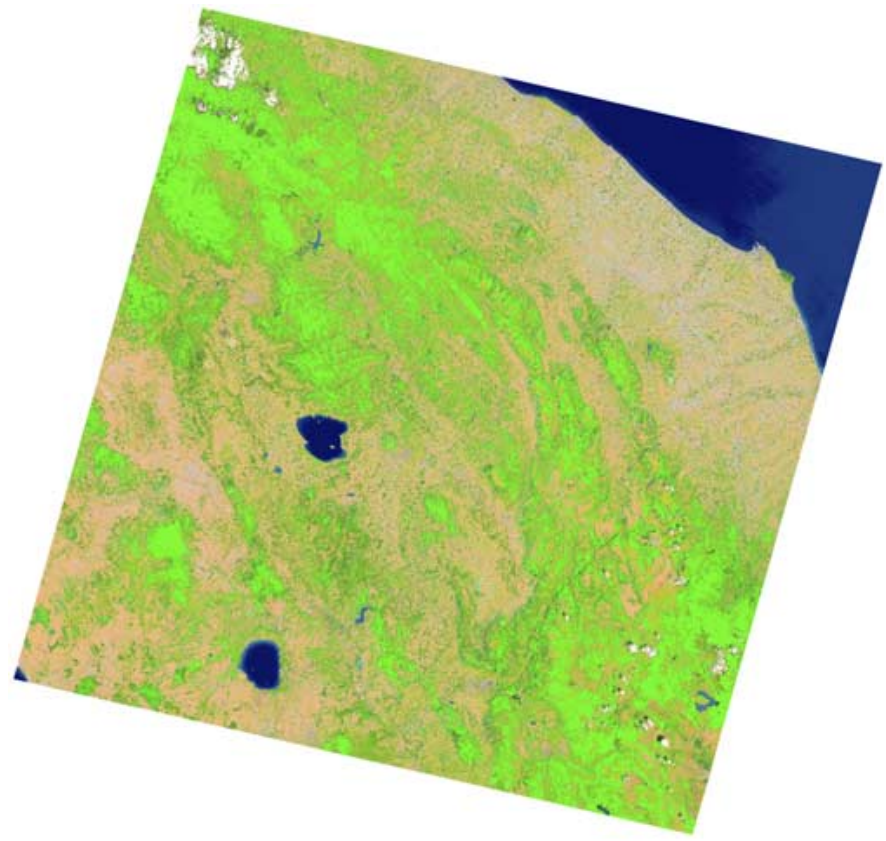

Fig. 9. Spectral rule-based system's output map generated from the ETM+ scene shown in Fig. 8. Vegetated surface types are in different shades of green, water bodies are in dark and light blue, and barren land and built-up candidate areas are brown and gray, respectively (for more details, refer to text).

image partition, $68 \%$ of the whole Landsat scene belongs to the vegetation candidate area (identified as $\mathrm{V}$ ) and $31.5 \%$ belongs to the nonvegetatation surface type (identified as NV). Only a small image percentage $(0.5 \%$, which belongs to spectral type $\mathrm{SU})$ is assigned to class unknown.

The accuracy of the vegetation/nonvegetation binary classification is assessed by means of a reference data set consisting of 500 testing samples located by means of a simple random sampling technique (i.e., where each pixel has an equal and an

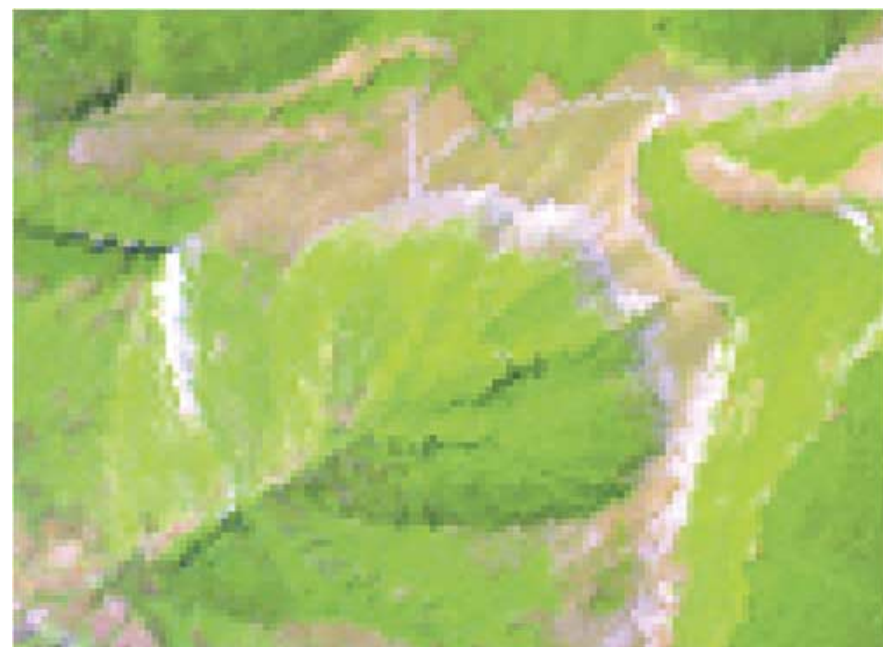

Fig. 10. Subset $(400 \times 300$ pixels in size $)$ of the Landsat-7 ETM+ false color composition image (R: TM5, G: TM4, B: TM1) shown in Fig. 8.

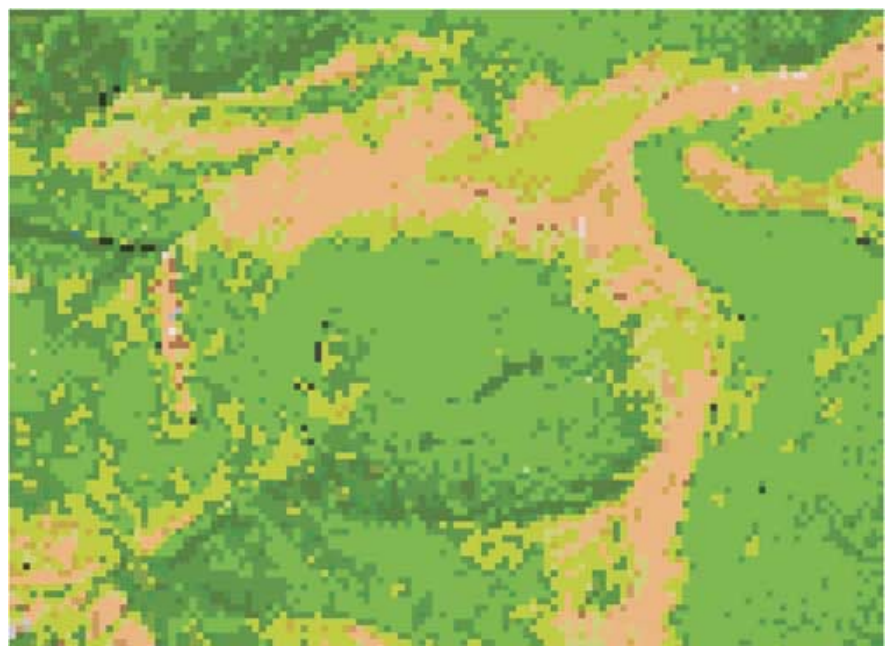

Fig. 11. Subset $(400 \times 300$ pixels in size $)$ of the spectral knowledge-based map shown in Fig. 9. Forest covers are classified as SV_SC (bright green), AV_SC (dark green), and SHV_LSC (very dark green). Pastures belong to the vegetated spectral type ASR_SC (okra). Bare soils belong to the nonvegetated spectral type BBB_SC (light brown).

independent chance of being selected; refer to [11]) on a mosaic of 46 PAN orthophotos acquired at $1-\mathrm{m}$ resolution during the Agea flight campaign in 1998, covering a surface area of approximately $2000 \mathrm{~km}^{2}$, which is approximately equivalent to a $28.5-\mathrm{m}$ resolution Landsat image of $1200 \times 2000$ pixels. These 500 random reference samples are labeled by means of a hierarchical visual analysis of the 28.5-m resolution MS Landsat ETM+ scene at hand in cascade to the 1-m resolution PAN orthophotos as described below (refer to Fig. 14).

First, 1-m resolution reference points are partitioned into pure-forest/agricultural fields' samples by means of a visual interpretation of the orthophotos based on the FAO-FRA2000 forest definition, such that ambiguous forest samples are excluded at this stage [44]. Second, 1-m resolution reference points are mapped onto the $28.5-\mathrm{m}$ resolution Landsat color scene. Finally, the $28.5-\mathrm{m}$ resolution reference data set is stratified into vegetation and nonvegetation pixels based on a visual analysis of the Landsat imagery. This visual analysis 


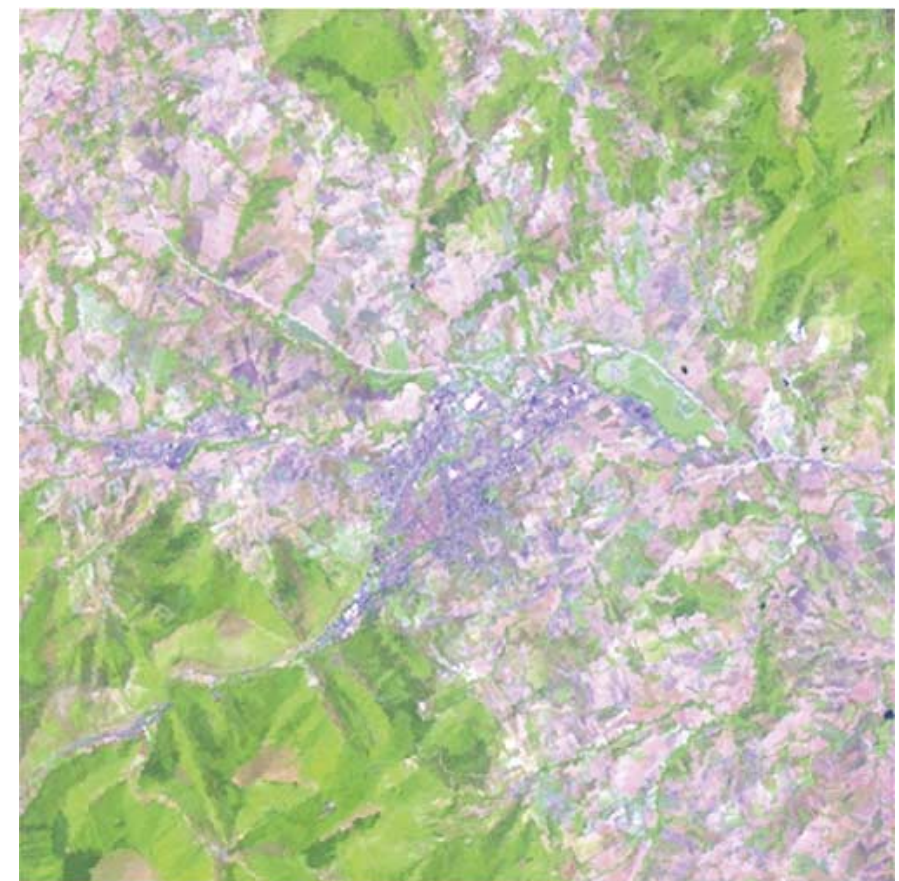

Fig. 12. Subset $(1200 \times 1200$ pixels in size $)$ of the Landsat-7 ETM+ false color composition image (R: TM5, G: TM4, B: TM1) shown in Fig. 8.

assigns 49 samples at $28.5-\mathrm{m}$ resolution out of the 278 forest reference examples at $1-\mathrm{m}$ resolution to class nonvegetation due to forest boundary effects in the coarse-resolution Landsat scene. Analogously, the 217 agricultural land reference samples are partitioned into $28.5-\mathrm{m}$ resolution vegetated or ploughed agricultural land samples. As a result, the final testing sample set comprises 398 vegetated samples and 102 nonvegetated samples at $28.5-\mathrm{m}$ spatial resolution.

Table X shows the distribution of reference samples among spectral types, where dark table cells correspond to misclassification occurrences (namely, three vegetated and six nonvegetated samples are wrongly mapped). Class-specific mapping accuracies, the classification OA and the Kappa coefficient, are reported in Table XI, where classification error tolerance estimates are computed according to (15) and (16) [11].

To summarize, based on both qualitative and quantitative results acquired over extended surface areas (namely, classification results are validated quantitatively across an area of $2000 \mathrm{~km}^{2}$, whereas qualitative validation by visual inspection holds for the whole Landsat scene covering $45000 \mathrm{~km}^{2}$ ), the tested spectral rule-based V/NV binary classification of a single-date Landsat imagery calibrated into planetary reflectance is considered accurate (e.g., OA equals $98.2 \% \pm$ $0.0 \%$ ) and robust against the presence of shadow areas and large within-class spectral variations. Such a first-stage preliminary V/NV binary classification product can be input to a secondstage forest area detector whose analysis focuses on the image subset V exclusively, which accounts for, say, $70 \%$ of the whole image area without requiring any user supervision.

\section{B. Landsat-5 TM}

A well-known strategy potentially capable of improving the accuracy of a purely supervised (e.g., MLP) classification of a very heterogeneous RS imagery (where reference data are

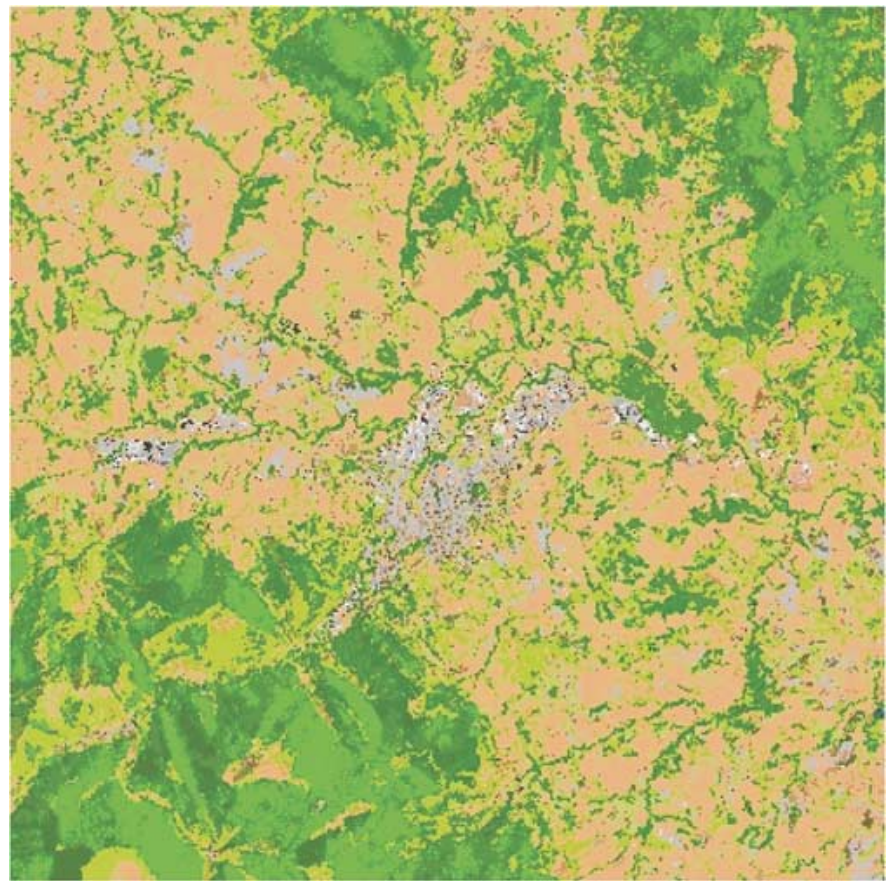

Fig. 13. Subset $(1200 \times 1200$ pixels in size $)$ of the spectral knowledge-based map shown in Fig. 9. Forest covers and agricultural fields are classified as SV_SC (bright green), AV_SC (dark green), or SHV_LSC (very dark green). Nonvegetated surfaces belong to the spectral type BBB_SC (light brown), whereas urban areas belong to spectral categories SBB_SC (dark gray) or ABB_SC (light gray).

TABLE IX

VEGETATION/NONVEGETATION BINARY AGGREGATION of SPECTRAL Surface TyPes

\begin{tabular}{|l|l|}
\hline $\begin{array}{l}\text { Vegetation/non vegetation } \\
\text { binary mapping }\end{array}$ & $\begin{array}{l}\text { Acronyms of kernel spectral categories combined with a } \\
\text { logical or operator }\end{array}$ \\
\hline Vegetation spectral types (V) & $\begin{array}{l}\text { SV_SC or AV_SC or WV_SC or SSR_SC or ASR_SC or } \\
\text { SHR_LSC or AHR_LSC or DR_LSC or SHV_LSC or } \\
\text { WE_LSC or WR_LSC }\end{array}$ \\
\hline $\begin{array}{l}\text { Nonvegetation spectral types } \\
\text { (NV) }\end{array}$ & $\begin{array}{l}\text { CL_SC or SNIC_SC or WASH_SC or BBB_SC or SBB_SC or } \\
\text { ABB_SC or DBB_SC or SHB_LSC or SHCL_LSC or } \\
\text { TWASHSN LSC or TWA LSC }\end{array}$ \\
\hline Unknown & EmptySU_LSC \\
\hline
\end{tabular}

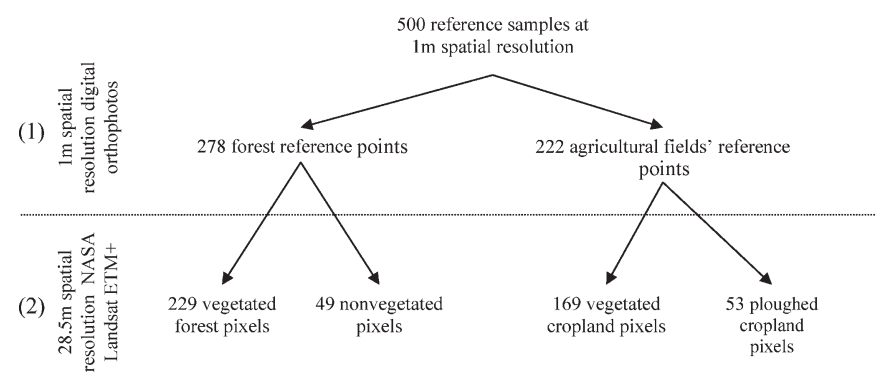

Fig. 14. Hierarchical classification of a reference data set based on a visual analysis of a 28.5-m-resolution MS Landsat ETM+ image in cascade to 1-mresolution PAN orthophotos.

difficult, tedious, or expensive to gather and, as a consequence, the unrepresentative sample problem is likely to occur [22]) is to employ two-stage hybrid data mapping techniques (see Section III-G). In line with this consideration, this section reports on a first-stage unsupervised kernel spectral strata detection system employed in cascade with a second-stage supervised MLP network classifier. 
TABLE $\mathrm{X}$

Distributions of TeSt SAMPLES AMONG SPECTRAL Surface TyPes. Dark TABle Cells Correspond to Mislabeled SAMPLE OCCURRENCES

\begin{tabular}{|c|c|c|c|c|c|}
\hline & \multirow[t]{2}{*}{ Spectral types } & \multicolumn{2}{|c|}{ Vegetated test samples } & \multicolumn{2}{|c|}{$\begin{array}{l}\text { Nonvegetated test } \\
\text { samples }\end{array}$} \\
\hline & & Forest & Agricultural & Forest & Agricultural \\
\hline \multirow{6}{*}{$\begin{array}{l}\text { Vegetatation } \\
\text { spectral types } \\
\text { (V) }\end{array}$} & SV SC & $54(10.8 \%)$ & $47(9.4 \%)$ & & \\
\hline & AV SC & $93(18.6 \%)$ & $54(10.8 \%)$ & & \\
\hline & SSR SC, SSR SC & $53(10.6 \%)$ & $45(9 \%)$ & & \\
\hline & SHR_LSC, AHR_LSC & $6(1.2 \%)$ & $2(0.4 \%)$ & & $1(0.2 \%)$ \\
\hline & $\begin{array}{l}\text { WV_LSC, DR_LSC, } \\
\text { WR_LSC }\end{array}$ & $22(4.4 \%)$ & $19(3.8 \%)$ & $2(0.4 \%)$ & $3(0.6 \%)$ \\
\hline & SHV LSC, WE LSC & & & & \\
\hline \multirow[t]{4}{*}{$\begin{array}{l}\text { Nonvegetatation } \\
\text { spectral types } \\
\text { (NV) }\end{array}$} & $\begin{array}{l}\text { BBB_SC, SBB_SC, } \\
\text { ABB_SC, DBB_LSC, } \\
\text { SHB_LSC }\end{array}$ & $1(0.2 \%)$ & $2(0.4 \%)$ & $47(9.4 \%)$ & $49(9.8 \%)$ \\
\hline & $\begin{array}{l}\text { WASH_SC, CL_SC, } \\
\text { SHCL_LSC, } \\
\text { SNIC_SC, } \\
\text { TWSHSN_LSC, } \\
\text { TWA_LSC }\end{array}$ & & & & \\
\hline & EmptySU_LSC & & & & \\
\hline & Overall percentage & \multicolumn{2}{|c|}{$398(79.6 \%)$} & \multicolumn{2}{|c|}{$102(20.4 \%)$} \\
\hline
\end{tabular}

TABLE XI

ACCURACIES OF THE SPECTRAL-RULE BASED VEGETATION/NONVEGETATION BINARY CLASSIFICATION

\begin{tabular}{|c|c|c|c|c|}
\hline Spectral type & $\begin{array}{c}\text { Test set } \\
\text { cardinality }\end{array}$ & $\begin{array}{c}\text { Class-specific } \\
\text { testing accuracy } \pm \\
\text { error tolerance } \delta_{i}\end{array}$ & $\begin{array}{c}\text { Testing OA } \pm \\
\text { error tolerance } \\
\delta\end{array}$ & $\begin{array}{c}\text { Testing Kappa } \\
\text { coefficient in [-1, } \\
1]\end{array}$ \\
\hline $\begin{array}{l}\text { Vegetation spectral } \\
\text { types (V) }\end{array}$ & 398 & $99.2 \pm 0.0 \%$ & \multirow{3}{*}{$98.2 \% \pm 0.0 \%$} & \multirow{3}{*}{0.94} \\
\hline $\begin{array}{l}\text { Nonvegetation } \\
\text { spectral types (NV) }\end{array}$ & 102 & $94.1 \pm 0.0 \%$ & & \\
\hline Tot & 500 & & & \\
\hline
\end{tabular}

The Landslide Early Warning (LEWIS) project (EU Contract EVG1-2001-00055) is considered a typical case of a poorly posed RS data classification problem. The goal of this project is to combine in-field information with multitemporal RS spaceborne image-understanding techniques to generate periodic "early warning maps" (updatable susceptibility maps) of landslide-prone areas where land cover/land use changes may lead to a decrease in slope stability. Two dates (spring and autumn) of 30-m resolution Landsat TM imagery are collected per year in the time period 1987-2000. Each pair of yearspecific images is potentially suitable for, first, discriminating agricultural fields from forest areas and, second, barren land from built-up land. Postclassification change information is obtained by comparing different year-specific thematic maps. Neither ground truth information nor information about changes in ground truth area is available. The exclusive source of reference data is a single May 1997 panchromatic orthophoto featuring a $1-\mathrm{m}$ spatial resolution and covering a tiny portion $(<5 \%)$ of the surface area of interest (centered on the village site of Caramanico in southern Italy), which is depicted by the Landsat TM images of the time series at hand (as an example, refer to the May 1987 image shown in Fig. 15).

A first purely supervised multitemporal TM data classification attempt conducted by nonexpert photointerpreters started from a visual interpretation of the 1997 black-and-white orthophoto where four target classes are identified as follows (refer to Table XII).

1) Arboreous. Due to difficulties in assessing different degrees of vegetation from panchromatic imagery, this class is thought to be a combination of forest land (USGS 4) plus rangeland featuring dense vegetation (USGS 3).

2) Agricultural land (USGS 2).
3) Urban or built-up land (USGS 1).

4) Barren land (USGS 7).

By means of additional time-consuming human supervision, ROIs belonging to these four target classes and visually estimated as affected by no thematic change through time are selected in the pair of Landsat images acquired for year 1987. The inductive learning classifier selected for this experiment is the very popular MLP network [15]. It is noteworthy that, despite the fact that the definition of a rejection rate is a wellknown objective of any classification training phase in the RS literature (e.g., refer to [9, p. 185]), in RS common practice, MLP networks are often applied without any outlier detection strategy, e.g., see [45]. This practice becomes acceptable if and only if the adopted classification scheme is mutually exclusive and totally exhaustive including class "unknown" [11], which is rarely the case (refer to Section I).

In the purely supervised classification experiment at hand, no outlier detection strategy is implemented. The selected MLP network architecture consists of 12 input nodes (corresponding to spectral channels in the stack of two TM images, excluding thermal channels) and four output nodes. The MLP hidden layer, which is selected by a model selection procedure, consists of 24 nodes, which is in line with recommendations found in [46]. To train and test the inductive learning MLP network, a holdout reference data resampling method is adopted. In particular, ROIs are randomly split into training and testing data sets according to proportions approximately equal to $2 / 3$ and $1 / 3$, respectively, in line with RS common practice [22].

Training (learning) accuracies, testing (generalization) accuracies, and error tolerances of the two-date four-class MLP classification experiment for year 1987 are reported in Table XII, where classification error tolerance estimates are computed according to (15) and (16). In this experiment, the training OA equals $93.6 \pm 0.5 \%$ with a Kappa coefficient equal to $0.87,{ }^{4}$ whereas the testing $\mathrm{OA}$ is $86.5 \% \pm 1.0 \%$ with a Kappa coefficient equals 0.72 .

As expected based on theoretical considerations, the twodate four-class supervised classification map generated by the induced MLP classifier employing no rejection rate is clearly affected by misclassification phenomena (namely, commission errors), e.g., cloud and snow pixels clearly visible in the May 1987 image (see Fig. 15) are assigned to barren land and builtup land, respectively. It is noteworthy that the removal of these transient thematic classes (which may be absent from one or more images of the multitemporal sequence) is not straightforward in a purely supervised multitemporal classification framework [48].

The operational conclusion of this purely supervised multitemporal classification attempt is that, due to the absence of ground truth data, complete and accurate classification of the 1987-2000 time series of Landsat-5 TM imagery is unaffordable in practice.

As a realistic alternative to purely supervised classification, the proposed system of prior knowledge-based rules is adopted to compute a sequence of single-date kernel spectral maps

\footnotetext{
${ }^{4}$ Coefficient Kappa $\in[-1,1]$ is.such that: a) $0<$ Kappa $<0.4$ represents poor agreement between mapping results and the reference data set, b) $0.4<$ Kappa $<0.8$ represents moderate agreement, and c) $0.8<$ Kappa $<1$ represents strong agreement [11]
} 


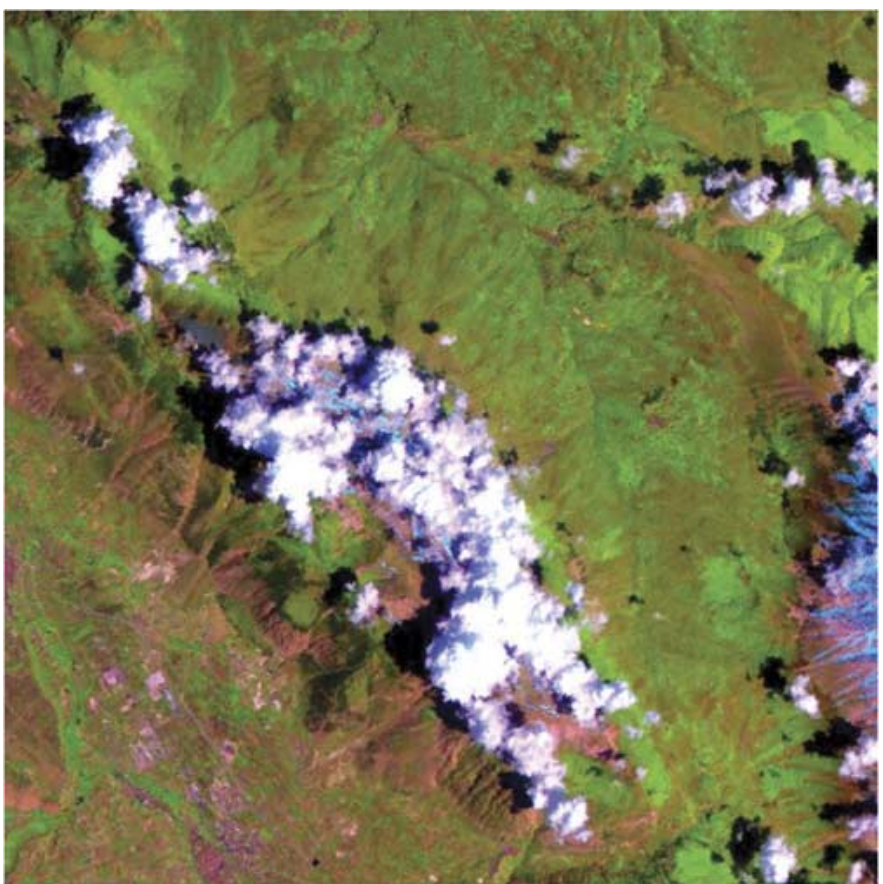

Fig. 15. Calibrated Landsat- 5 TM image $(400 \times 580$ pixels $)$, in false colors (R: band 5, G: band 4, B: band 1). Depicted area: Abruzzi region, Italy. Acquisition date: May 26, 1987.

TABLE XII

Two-Date Four-Class Classification Problem. Training and Testing Data Sets and Classification ACCuracies

\begin{tabular}{|c|c|c|c|c|c|}
\hline Class & Train & Test & $\begin{array}{c}\text { Class-specific } \\
\text { testing accuracy } \\
\pm \text { error tolerance } \\
\delta_{i} \\
\end{array}$ & $\begin{array}{l}\text { Testing OA } \\
\quad \pm \text { error } \\
\text { tolerance } \delta\end{array}$ & $\begin{array}{c}\text { Testing Kappa } \\
\text { coefficient in [-1, } \\
1]\end{array}$ \\
\hline 1. Arboreous & 6542 & 1847 & $90.7 \pm 0.2 \%$ & \multirow{5}{*}{$86.5 \% \pm 1.0 \%$} & \multirow{5}{*}{0.72} \\
\hline 2. Agricultural land & 729 & 334 & $74.8 \pm 0.7 \%$ & & \\
\hline 3. Urban or built-up land & 1106 & 121 & $69.4 \pm 1.3 \%$ & & \\
\hline 4. Barren land & 1156 & 241 & $79.7 \pm 0.8 \%$ & & \\
\hline Tot. & 9533 & 2543 & & & \\
\hline
\end{tabular}

from the input image time series without requiring any user supervision. As an example of a single-date kernel spectral map, let us consider the map in Fig. 16, which is generated from the single-date May 1987 TM image shown in Fig. 15. In Fig. 16, kernel spectral categories are depicted in line with Fig. 9 (refer to Section V-A).

A visual (qualitative) comparison of Fig. 16 with the input raw image shown in Fig. 15 reveals that transient classes, such as snow, clouds, and shadow areas, which are easy to detect visually, are labeled correctly. More fuzzy vegetation spectral categories like SSR_SC, ASR_SC, SHR_LSC, AHR_LSC, and DR_LSC are validated by means of a visual comparison of Fig. 16 with the May 1997 orthophoto. Although based on qualitative evidence, our conclusion is that the spectral preliminary map shown in Fig. 16 appears to be a viable source of reliable ground truth information.

Supported by this conclusion, one more single-date kernel spectral category map is extracted from the second Landsat imagery of the 1987 input pair, namely, the raw image acquired on September 1987. ROIs originally selected for the two-date four-class purely supervised classification problem are now revised to be consistent with the two single-date preliminary spectral maps and with a visual assessment of the 1997 or-

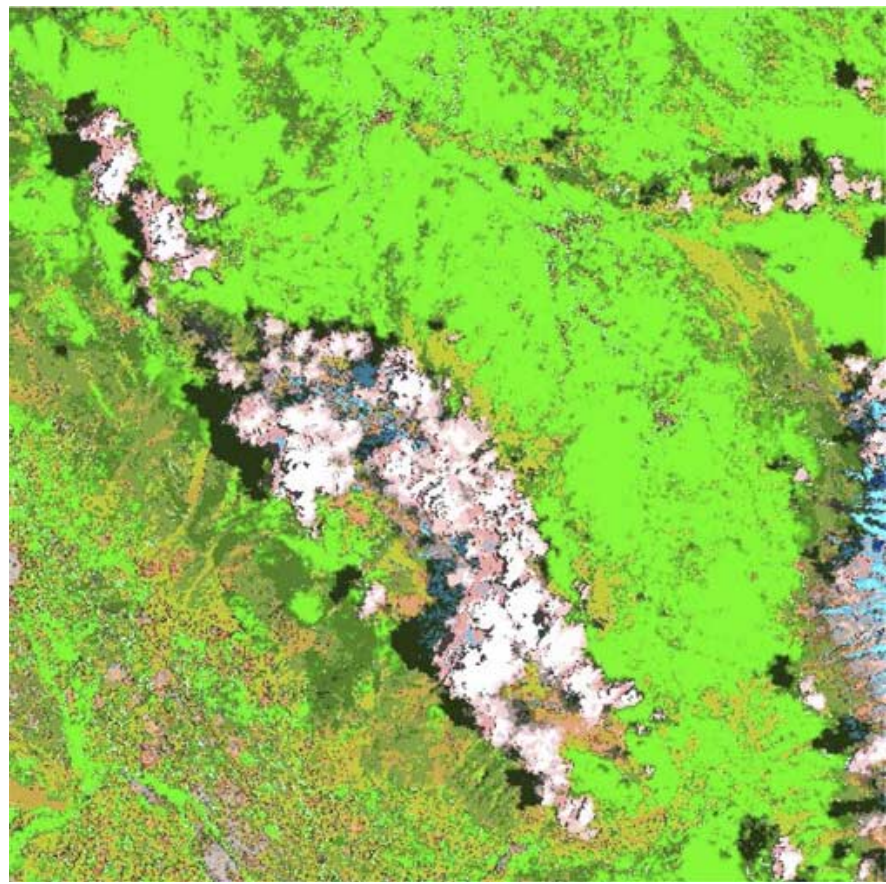

Fig. 16. Single-date kernel spectral category map generated by the spectral rule-based system from the calibrated May 1987 Landsat TM image shown in Fig. 15. Kernel spectral categories are depicted in line with Fig. 9 (refer to Section V-A for more details).

TABLE XIII

Five-Class Supervised Classification Problem. Classification ACCURACIES AND ERROR TOLERANCE UPON TRAINING AND TESTING DATA SETS

\begin{tabular}{|l|c|c|c|c|c|}
\hline \multicolumn{1}{|c|}{ Class } & Train & Test & $\begin{array}{c}\text { Class-specific } \\
\text { testing accuracy } \\
\pm \text { error } \\
\text { tolerance } \delta_{i}\end{array}$ & $\begin{array}{c}\text { Testing OA } \\
\text { error } \\
\text { tolerance } \delta\end{array}$ & $\begin{array}{c}\text { Testing Kappa } \\
\text { coefficient in [-1, } \\
\text { 1] }\end{array}$ \\
\cline { 1 - 4 } 1. Forest land & 1335 & 968 & $95.7 \pm 0.2 \%$ & & \\
\cline { 1 - 3 } 2. Agricultural land & 1260 & 1031 & $69.7 \pm 0.4 \%$ & & \multirow{2}{*}{0.75} \\
\cline { 1 - 3 } 3. Urban or built-up land & 405 & 338 & $84.3 \pm 0.6 \%$ & & \\
\cline { 1 - 3 } 4. Barren land & 404 & 208 & $88.5 \pm 0.7 \%$ & & \\
\cline { 1 - 3 } 5. Rangeland & 1212 & 646 & $72.7 \pm 0.5 \%$ & & \\
\hline
\end{tabular}

thophoto. Starting from spectral categories SSR_SC, ASR_SC, SHR_LSC, AHR_LSC, and DR_LSC found in the pair of single-date kernel spectral maps, ROIs belonging to a new class, which is identified as class "rangeland," are localized in the input May-September 1987 raw image pair. Next, a new fiveclass MLP classifier is trained based on the revised reference data set. After training convergence is reached, the generalization capability of the five-class MLP network is assessed according to a holdout reference data resampling method. In this case, testing results are shown in Table XIII. A comparison between Tables XII and XIII reveals that the five-class MLP classification accuracy improves the four-class MLP classification results with regard to three out of four classes. This observation confirms the utility of a hybrid learning strategy (in this example, prior knowledge-based in cascade with supervised data-driven) versus purely supervised learning strategies in a real-world RS image data mapping problem (refer to Section I).

Despite quantitative results shown in Table XIII, the overall (image-wide) mapping accuracy of the five-class output map is poor due to the presence of FP samples as the adopted classification scheme is not totally exhaustive (e.g., snow and 
cloud pixels are assigned to classes built-up and barren land, respectively). To introduce a simple outlier detection strategy (required to make any supervised RS data learning phase effective; see [9, p. 185]), a so-called strict generalization is pursued while ignoring the problem of input samples lying on the overlapping region between two or more classes (refer to Section III-G2). In particular, a sequence of simple oneclass classifiers (refer to Section III-G2) is implemented as follows.

1) Cluster every class-specific training data set independently (e.g., by means of a standard $k$-means clustering algorithm).

2) For each class and every cluster belonging to that class, compute the cluster's standard deviation as the standard deviation of the class-specific training samples falling in the cluster's Voronoi polyhedron (receptive field, equivalent to the set of points in feature space whose Euclidean distance from that cluster's barycenter is minimum).

3) Exclude from further mapping (i.e., consider as outliers) any input pattern that falls outside of a volume in input space that wraps around each cluster's barycenter $\pm q$ standard deviations (typically, $q$ equals 2 or 3).

In this application example, the outlier rejection free parameter $q$ is user-defined by means of a trial-and-error optimization strategy. First, the temporal sequence of the single-date kernel spectral maps is adopted as a quality ground truth data set. Second, the rejection rate is adjusted until input samples belonging to kernel spectral categories CL_SC, SNIC_SC, WASH_SC, SHV_LSC, SHB_LSC, SHCL_LSC, TWASHSN_LSC, and TWA_LSC, which are not represented in the (nonexhaustive) target set of supervised classes (namely, "forest," "agricultural land," "urban land," "barren land," and "rangeland"), are effectively rejected. The result of this outlier rejection strategy is clearly visible in Fig. 17, where rejected input samples are depicted in black (e.g., pixels belonging to kernel spectral types clouds and snow are masked out effectively). In line with theory (see Section III-G2), such a simple novelty detection strategy reduces, but does not eliminate, misclassification occurrences due to the presence of input samples lying on the overlapping region between two or more classes (e.g., agricultural land is overestimated against supervised classes forest and rangeland according to kernel spectral categories adopted as the ground truth data set).

\section{CONCLUSION}

This paper presents an original prior spectral knowledgebased context-insensitive (per-pixel) system of (either crisp or fuzzy) decision rules designed for mapping calibrated Landsat TM and ETM+ images into a discrete finite set of kernel spectral categories.

The degree of user supervision required to detect kernel spectral categories (which is null) is the same as unsupervised data clustering and far inferior to reference sample selection required by supervised data learning classifiers. The symbolic meaning (level of abstraction) of kernel spectral categories is intermediate between those of clusters and segments (which is null) and that of land cover classes.

From a theoretical standpoint, in a two-stage hybrid data learning framework suitable for dealing with the large spa-

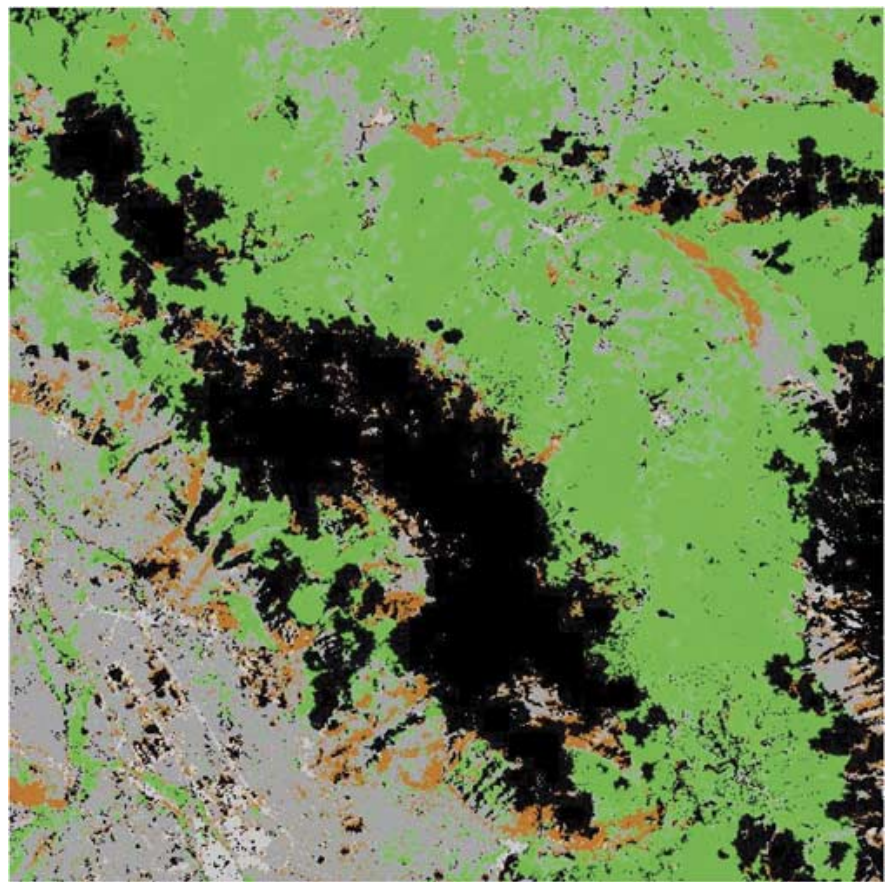

Fig. 17. Two-date five-class purely supervised classification map generated by an MLP classifier without rejection rate from two dates of Landsat-5 TM imagery acquired in May (see Fig. 15) and September 1987, followed by an outlier rejection step. MLP detected classes are: forest land (bright green), agricultural land (dark gray, clearly overstimated), built-up land (in light gray, erroneously assigned to shadow areas), barren land (brown, erroneously assigned to clouds), and rangeland (dark green). In cascade to purely supervised classification, a strict generalization stage is employed to reject novel input patterns, which are shown in black. In line with theoretical expectations, outliers mainly belong to thematic classes not implemented in the adopted five-class classification scheme, namely, snow, clouds, and shadow areas.

tial and spectral complexity characterizing RS imagery, the proposed purely spectral mapping system can be conceived as an unsupervised learning first stage (where no reference sample is required) to be employed in cascade with stratified (i.e., category-, class-, and application domain-specific) data clustering, image segmentation, and classification second-stage algorithms (exploiting context-sensitive mapping criteria such as spatial, contextual, geometric, and textural properties of target land cover classes).

The potential utility of the proposed rule-based system is tested in two experiments where the classification of Landsat imagery depicting extended surface areas is provided with little or no ground truth knowledge. Both experiments prove that kernel spectral maps can be considered a reliable source of prior spectral knowledge useful in a variety of RS data understanding applications such as vegetation/nonvegetation binary mapping, stratified classification, and outlier detection.

Designed for calibrated Landsat imagery, the proposed system of rules is easily scalable to other high and medium spatial-resolution spaceborne optical sensors like SPOT-4 and -5 (either one featuring no TIR channel) and ASTER.

Vice versa, the spectral resolution characterizing the very popular VHR spaceborne optical sensors like Ikonos and QuickBird, which feature neither MIR nor TIR channel, makes the proposed spectral knowledge-based classification scheme unable to provide a complete and reliable RS image binary partition into the two basic kernel categories, i.e., vegetation 
and nonvegetation (refer to Fig. 2). This conclusion is supported by the additional experimental results gathered from existing literature [39]. It is also theoretically supported by wellknown vegetation-specific spectral properties requiring conditions (Vis $\ll N I R$ ) and (NIR $\gg$ MIR), which are equivalent to conditions (NDVI is high) and (NDBSI is low) [which exclude pixels belonging to class rangeland (refer to the proposed system of rules)]. Two corollaries stem from this conclusion.

1) In a fully automatic spectral knowledge-based classification framework, NDVI is clearly unable, both theoretically and experimentally, to guarantee a complete and reliable vegetation/nonvegetation image partition as this index alone is unfeasible to highlight subtle differences in canopy density (like those characterizing the USGS land cover class rangeland), as clearly acknowledged in some existing RS literature (e.g., [37]). Irrespective of the classification system at hand, binary discrimination between classes vegetation and nonvegetation requires at least a pair of indexes, e.g., NDVI and NDBSI, to guarantee separability of these two land cover types in feature space.

2) The spectral performance and radiometric requirements (band centers and spectral widths) of the existing VHR spaceborne optical sensors, which are supporting neither MIR nor TIR bands, appear clearly unsuitable for supporting fully automatic prior spectral knowledge-based image classification systems. Rather, VHR data classification systems must heavily rely upon supervised data classification approaches based on (expensive, difficult to gather) reference data sets.

A final remark regards recent experimental statistics gathered in ongoing applications of the proposed prior knowledge-based system. These statistics appear to indicate that thresholds involved in the definition of FSs listed in Table III are invariant to changes in the geographic position of the calibrated input image (as anticipated in Section III-D). This important operational development would further increase the usability of the proposed system without decreasing its adaptivity to changing input data sets.

Future developments of the proposed spectral rule-based classifier will focus on stratified segmentation and classification to extract cropland from vegetated candidate areas and bare soil cropland from nonvegetated candidate areas based on geometric properties such as segment compactness and straightness of segment boundaries, in line with [2].

\section{ACKNOWLEDGMENT}

The authors would like to thank F. De Natale and F. Giannetti for providing them with part of the reference data employed for classification accuracy assessment, and the associate editor and anonymous reviewers for their helpful comments.

\section{REFERENCES}

[1] J. Ton, J. Sticklen, and A. K. Jain, "Knowledge-based segmentation of Landsat images," IEEE Trans. Geosci. Remote Sens., vol. 29, no. 2, pp. 222-231, Mar. 1991.

[2] M. Nagao and T. Matsuyama, A Structural Analysis of Complex Aerial Photographs. New York: Plenum, 1980.
[3] A. Baraldi and F. Parmiggiani, "A Nagao-Matsuyama approach to high resolution satellite image classification," IEEE Trans. Geosci. Remote Sens., vol. 32, no. 4, pp. 749-758, Jul. 1994.

[4] A. Bardossy and L. Samaniego, "Fuzzy rule-based classification of remotely sensed imagery," IEEE Trans. Geosci. Remote Sens., vol. 40, no. 2, pp. 362-374, Feb. 2002.

[5] E. Binaghi, P. Madella, M. G. Montesano, and A. Rampini, "Fuzzy contextual classification of multisource remote sensing images," IEEE Trans. Geosci. Remote Sens., vol. 35, no. 2, pp. 326-340, Mar. 1997.

[6] R. Sugumaran, M. K. Pavuluri, and D. Zerr, "The use of high-resolution imagery for identification of urban climax forest species using traditional and rule-based classification approach," IEEE Trans. Geosci. Remote Sens., vol. 41, no. 9, pp. 1933-1939, Sep. 2003.

[7] G. Mehldau and R. A. Schowengerdt, "A C-extension for rule-based image classification systems," Photogramm. Eng. Remote Sens., vol. 56, no. 6, pp. 887-892, Jun. 1990.

[8] M. Avci and Z. Akyurek, A Hierarchical Classification of Landsat TM Imagery for Landcover Mapping. Istanbul, Turkey: ISPRS, Jul. 12-23, 2004.

[9] P. H. Swain and S. M. Davis, Remote Sensing: The Quantitative Approach. New York: McGraw-Hill, 1978.

[10] [Online]. Available: htpp://ltpwww.gsfc.nasa.gov/IAS/handbook/ handbook_htmls/chapter11/chapter11.html

[11] R. G. Congalton and K. Green, Assessing the Accuracy of Remotely Sensed Data. Boca Raton, FL: Lewis, 1999.

[12] [Online]. Available: http://asterweb.jpl.nasa.gov

[13] D. Marr, Vision. New York: Freeman, 1982.

[14] P. Blonda, V. la Forgia, G. Pasquariello, and G. Satalino, "Feature extraction and pattern classification of remote sensing data by a modular neural system," Opt. Eng., vol. 35, no. 2, pp. 536-542, Feb. 1996.

[15] C. M. Bishop, Neural Networks for Pattern Recognition. Oxford, U.K.: Clarendon, 1995.

[16] G. Buttner, T. Hajos, and M. Korandy, "Improvements to the effectiveness of supervised training procedures," Int. J. Remote Sens., vol. 10, no. 6, pp. 1005-1013, 1989.

[17] C. Leprieur, J. M. Durand, and J. L. Peyron, "Influence of topography on forest reflectance using Landsat thematic mapper and digital terrain data," Photogramm. Eng. Remote Sens., vol. 54, no. 4, pp. 491-496, Jun. 1988.

[18] L. Bruzzone and F. Prieto, "An adaptive semiparametric and contextbased approach to unsupervised change detection in multitemporal remote sensing images," IEEE Trans. Image Process., vol. 11, no. 4, pp. 452-466, Apr. 2002.

[19] [Online]. Available: http://earth.esa.int/rootcollection/sysutil/00699.html

[20] V. Cherkassky and F. Mulier, Learning From Data: Concepts, Theory, and Methods. New York: Wiley, 1998.

[21] T. Mitchell, Machine Learning. New York: McGraw-Hill, 1997.

[22] A. Baraldi, L. Bruzzone, and P. Blonda, "Quality assessment of classification and cluster maps without ground truth knowledge," IEEE Trans. Geosci. Remote Sens., vol. 43, no. 4, pp. 857-873, Apr. 2005.

[23] L. Delves, R. Wilkinson, C. Oliver, and R. White, "Comparing the performance of SAR image segmentation algorithms," Int. J. Remote Sens., vol. 13, no. 11, pp. 2121-2149, 1992.

[24] S. Zucker, "Region growing: Childhood and adolescence," Comput. Graph. Image Process., vol. 5, no. 2, pp. 382-399, 1976.

[25] T. N. Pappas, "An adaptive clustering algorithm for image segmentation," IEEE Trans. Signal Process., vol. 40, no. 4, pp. 901-914, Apr. 1992.

[26] P. Perona and J. Malik, "Scale-space and edge detection using anisotropic diffusion," IEEE Trans. Pattern Anal. Mach. Intell., vol. 12, no. 7, pp. 629-639, Jul. 1990.

[27] A. Mojsilovic, J. Kovacevic, J. Hu, R. Safranek, and S. K. Ganapathy, "Matching and retrieval based on the vocabulary and grammar of color patterns," IEEE Trans. Image Process., vol. 9, no. 1, pp. 38-54, Jan. 2000.

[28] R. Duin, "Compactness and complexity of pattern recognition problems," in Proc. Int. Symp. Pattern Recog., 1999, pp. 124-128.

[29] Y. Pao, Adaptive Pattern Recognition and Neural Networks. Reading, MA: Addison-Wesley, 1989.

[30] B. Fritzke, "Incremental neuro-fuzzy systems," in Proc. SPIEApplications Soft Computing Conf., San Diego, CA, Jul. 27-Aug. 1, 1997. [Online]. Available: http://www.ki.inf.tu-dresden.de/ fritzke/ FuzzyPaper/t.html

[31] D. Chakraborty and N. R. Pal, "A neuro-fuzzy scheme for simultaneous feature selection and fuzzy rule-based classification," IEEE Trans. Neural Netw., vol. 15, pp. 110-123, Jan. 2004.

[32] O. Tobias and R. Seara, "Image segmentation by histogram thresholding using fuzzy sets," IEEE Trans. Image Process., vol. 11, no. 12, pp. 14571465, Dec. 2002. 
[33] IDL User Manual, Research Systems, Inc., Boulder, CO, 2005.

[34] eCognition User Guide, Definiens Imaging GmbH, Munich, Germany, 2005.

[35] D. C. Burr and M. C. Morrone, "A nonlinear model of feature detection," in Nonlinear Vision: Determination of Neural Receptive Fields, Functions, and Networks, R. B. Pinter and N. Bahram, Eds. Baca Raton, FL: CRC, 1992, pp. 309-327.

[36] C. Perry, J. Lautenschlager, and L. Lautenschlager, "Functional equivalence of spectral vegetation indices," J. Remote Sens. Environ., vol. 14, pp. $170-182,1984$

[37] P. Roy, S. Miyatake, and A. Rikimaru. (1997). Biophysical Spectral Response Modeling Approach for Forest Density Stratification [Online]. Available: http://www.gisdelopment.net/aars/acrs/1997/ tTM5/tTM5008a.shtml

[38] R. Irish, "Landsat 7 automatic cloud cover assessment (ACCA)," in Proc. SPIE-Algorithms Multispectral, Hyperspectral, and Ultraspectral Imagery VI, S. S. Shen and M. R. Descour, Eds., 2000, vol. 4049, pp. 348-355. [Online]. Available: 1tpwww.gsfc.nasa.gov/IAS/handbook/ pdfs/ACCA_SPIE_paper.pdf

[39] Sarmap Final Report, DUP 2000: Mapping of Clear Cuts With SAR for Illicit Crop Monitoring in Colombian Forest, 2001, Angers, France: ESA. AO/1-3585/00I-IW.

[40] C. Huang, B. Wylie, L. Yang, C. Homer, and G. Zylstra, "Derivation of a tasselled cap transformation based on Landsat 7 planetary reflectance," Int. J. Remote Sens., vol. 23, no. 8, pp. 1741-1748, 2002.

[41] J. Horne, "A tasseled cap transformation for Ikonos images," in Proc. ASPRS Conf., Anchorage, AK, May 2003. [Online]. Available: http://www.spaceimaging.com/whitepapers_pdfs/2003/A\%20Tasseled\% 20 Cap $\% 20$ Transformation \% 20for \% 20IKONOS \% 20Images-ASPRS \% 202003.pdf

[42] [Online]. Available: http://inforest.jrc.it/activities/biodiversity

[43] C. M. Estreguil, P. Vogt, M. Cerruti, and M. Maggi, "JRC contribution to reporting needs of EC nature and forest policies," in Monitoring and Indicators of Forest Biodiversity in Europe. From Ideas to Operationality, M. Marchetti, Ed. Joensuu, Finland: Eur. Forest Inst., 2004, pp. 91-102.

[44] FAO, FRA 2000-Terms and Definitions. FRA Working Paper 1, 1998. [Online]. Available: http://www.fao.org/forestry/fo/fra/index.jsp

[45] W. Zhou, "Verification of the nonparametric characteristics of back propagation neural networks for image classification," IEEE Trans. Geosci. Remote Sens., vol. 37, no. 2, pp. 771-779, Mar. 1999

[46] I. Kanellopoulos and G. G. Wilkinson, "Strategies and best practice for neural network image classification," Int. J. Remote Sens., vol. 18, no. 4, pp. 711-725, 1997.

[47] E. Lunetta and C. Elvidge, Remote Sensing Change Detection: Environmental Monitoring Methods and Applications. London, U.K.: Taylor \& Francis, 1999.

[48] P. A. Brivio, M. Maggi, E. Binaghi, and I. Gallo, "Mapping burned surfaces in sub-Saharan Africa based on multi-temporal neural classification," Int. J. Remote Sens., vol. 24, no. 20, pp. 4003-4016, Oct. 2003.

[49] C. Small, "Multiresolution analysis of urban reflectance," in Proc. IEEE/ ISPRS Workshop Remote Sens. and Data Fusion Urban Areas, Rome, Italy, 2001, pp. 15-19, Paper 23.

[50] [Online]. Available: http://www.gisdevelopment.net/aars/acrs/1997/tTM5/ tTM5008a.shtml

[51] [Online]. Available: http://www.uswcl.ars.ag.gov/epd/remsen/Vi/VIworks $\mathrm{htm}$

[52] M. Herold, M. Gardner, V. Noronha, and D. Roberts, Spectrometry and Hyperspectral Remote Sensing of Urban Road Infrastructure. [Online]. Available: http://satjournal.tcom.ohiou.edu/pdf/herold.pdf

[53] A. Asmat, S. Mansor, and W. T. Hong, "Rule based classification for urban heat island mapping," in Proc. 2nd FIG Regional Conf., Marrakech, Morocco, Dec. 2-5, 2003. [Online]. Available: http:// www.fig.net/pub/morocco/proceedings/TS18/TS18_4_asmat_et_al.pdf

[54] M. Markou and S. Singh, "Novelty detection: A review-Part 1: Statistical approaches," Signal Process., vol. 83, no. 12, pp. 2481-2497, Dec. 2003.

[55] — - "Novelty detection: A review—Part 2: Neural network-based approaches," Signal Process., vol. 83, no. 12, pp. 2499-2521, Dec. 2003

[56] E. Pekalska, D. Tax, and R. Duin, "One-class LP classifier for dissimilarity representation," in Advances in Neural Information Processing Systems, S. Becker, S. Thrun, and K. Obermayer, Eds. Cambridge, MA: MIT Press, 2003.

[57] D. Tax and R. Duin, "Uniform object generation for optimizing one-class classifiers," J. Mach. Learn. Res., vol. 2, pp. 155-173, 2001.

[58] D. Chakraborty and N. R. Pal, "A novel training scheme for multilayered perceptrons to realize proper generalization and incremental learning," IEEE Trans. Neural Netw., vol. 14, no. 1, pp. 1-14, Jan. 2003.
[59] [Online]. Available: http://www.mlahanas.de/MOEA/Med/ROC21.htm

[60] S. Geman and D. Geman, "Stochastic relaxation, Gibbs distribution, and the Bayesian restoration of images," IEEE Trans. Pattern Anal. Mach. Intell., vol. PAMI-6, no. 6, pp. 721-741, Nov. 1984.

[61] U.S. Geological Survey, Landsat (Sensor: ETM+) Scene, WRS-(2 or 1), Path (191), Row (030), Level (Orthorectified), Sioux Falls, SD: USGS. [Online]. Available: http://www.landcover.org

[62] ERDAS Imagine 8.7-User Manual.

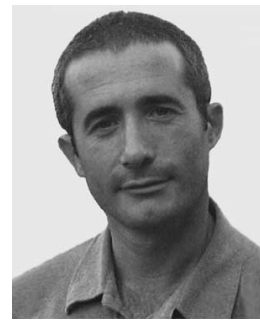

Andrea Baraldi was born in Modena, Italy, in 1963 He received the laurea (M.S.) degree in electronic engineering from the University of Bologna, Bologna, Italy, in 1989.

From 1989 to 1990, he was a Research Associate with CIOC-National Research Council (CNR), Bologna, and served in the army at the Instituto Geografico Militare, Florence, Italy, where he worked on satellite image classifiers and geographic information systems (GIS). From 1991 to 1993, he was a Consultant at ESA-ESRIN, Frascati, Italy, where he worked on object-oriented applications for GIS. From December 1997 to June 1999, he was a Postdoctoral Fellow in artificial intelligence at the International Computer Science Institute, Berkeley, CA. From 2000 to 2002, he was with the GVM-IES unit of the European Commission Joint Research Centre (JRC) Ispra, Italy, as a Postdoctoral Researcher, where he worked on the development and validation of classification algorithms for continental-scale mapping of radar mosaics of forest ecosystems. He is currently with the Institute for the Protection and Security of the Citizen-Support to External Security unit of the JRC, involved with optical and radar image interpretation for terrestrial surveillance and change detection. Since his master thesis he has continued his collaboration with ISAC-CNR in Bologna and ISSIA-CNR in Bari, Italy. His main interests center on image segmentation and classification, with special emphasis on multiscale texture analysis and neural network applications in computer vision.

Dr. Baraldi has been serving as an Associate Editor of the IEEE TRANSACTIONS ON NEURAL NETWORKS since 2001.

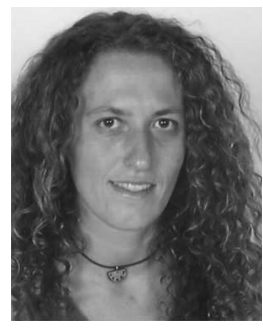

Virginia Puzzolo received the laurea (M.S.) degree in natural science and the Ph.D. degree in plant ecology and biology in Mediterranean environment from the University of Catania, Catania, Italy, in 1997 and 2002, respectively.

She was a Research Fellow sponsored by the Bonino-Puleio Foundation of Messina, Italy, and worked on the development of a phytoclimatic classification of eastern Sicily vegetation using a multitemporal data set of Landsat images at the Plan Biology Department, "Federico II" University, Naples, Italy. From December 1999 to May 2000, she was with the National Research Council, formerly CNR-IATA, in Florence, Italy. From December 2000 to May 2001, she was with the EGEO Unit of the ex-Space Applications Institute of the Joint Research Centre (JRC), Ispra, Italy. From January to September 2002, she was a Marie Curie Fellow at the "CULTural LANDscapes of Europe," Marie Curie Training Sites, University of Bergen, Bergen, Norway. After receiving the Ph.D. degree, she was a temporary Research Assistant for two years with the Research Forest Management Institute, Trento, Italy, where she worked on the applications of remotely sensed optical images for forest classification and landscape analysis in rugged areas. She was also a Research Fellow sponsored by the Foreign Italian Ministry and worked on testing the application of a $k$-nearest neighbor classification method for the estimation of forest structural parameter in Mediterranean forests at the Finnish Forest Research Institute, Helsinki, Finland. She recently joined the Institute for Environment and Sustainability of JRC, as a Postdoctoral Researcher. Her previous research interests include ecological and structural analysis of the Mt. Etna forests based on an integration of remotely sensed optical images and field data. Her current research interests are the generation of a forest cover classification map at a Pan-European scale from Landsat imagery in the framework of the INFOREST Action of the Land Management Unit and the assessment of forest cover changes and forest spatial pattern changes from late 1980s to 2000 in the Mediterranean region with associated effects on biodiversity. 


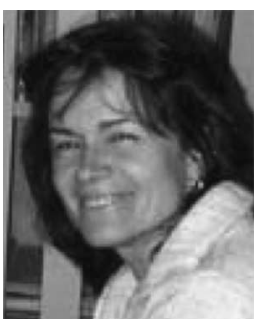

Palma Blonda received the Ph.D. degree in physics from the University of Bari, Bari, Italy, in 1980.

In 1984, she joined the Institute of Studies on Intelligent Systems for Automation (formerly Institute for Signal and Image Processing) at the Italian National Research Council (CNR), in Bari. She was recently involved with the project "Landslide Early Warning Integrated System (LEWIS)" (EVG1-CT2001-00055), which was founded by the European Community in the framework of the Fifth PQ, where she worked on the application of multisource data integration and classification techniques for the extraction of Earth observation detectable superficial changes of landslide-related factors to be used in earlywarning mapping. Her research interests include digital image processing, fuzzy logic and neural networks, soft computing applied to the integration, and classification of multisource remote-sensed data.

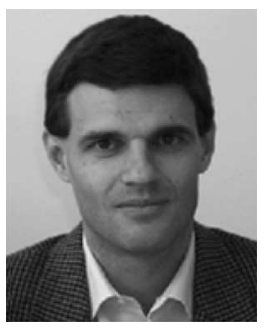

Lorenzo Bruzzone (S'95-M'98-SM'03) received the laurea (M.S.) degree in electronic engineering (summa cum laude) and the Ph.D. degree in telecommunications from the University of Genoa, Genoa, Italy, in 1993 and 1998, respectively.

From 1998 to 2000, he was a Postdoctoral Researcher at the University of Genoa. From 2000 to 2001, he was an Assistant Professor at the University of Trento, Trento, Italy, and from 2001 to 2005 , he was an Associate Professor at the same university. Since March 2005, he has been a Full Professor of telecommunications at the University of Trento, where he currently teaches remote sensing, pattern recognition, and electrical communications. He is currently the Head of the Remote Sensing Laboratory in the Department of Information and Communication Technology, University of Trento. His current research interests are in the area of remote-sensing image processing and recognition (analysis of multitemporal data, feature selection, classification, regression, data fusion, and machine learning). He conducts and supervises research on these topics within the frameworks of several national and international projects. Since 1999, he has been appointed Evaluator of project proposals for the European Commission. He is the author (or coauthor) of more than 150 scientific publications, including journals, book chapters, and conference proceedings. He is a Referee for many international journals and has served on the Scientific Committees of several international conferences.

Dr. Bruzzone ranked first place in the Student Prize Paper Competition of the 1998 IEEE International Geoscience and Remote Sensing Symposium (Seattle, July 1998). He was a recipient of the Recognition of IEEE TRANSACTIONS ON Geoscience ANd Remote SENSING Best Reviewers in 1999 and was a Guest Editor of a Special Issue of the IEEE TRANSACTIONS ON GEOSCIENCE AND REMOTE SENSING on the subject of the analysis of multitemporal remote-sensing images (November 2003). He was the General Chair and Cochair of the First and Second IEEE International Workshop on the Analysis of Multi-temporal Remote-Sensing Images. Since 2003, he has been the Chair of the SPIE Conference on Image and Signal Processing for Remote Sensing. $\mathrm{He}$ is an Associate Editor of the IEEE TRAnsactions on GeOsCIENCE AND Remote Sensing. He is a member of the Scientific Committee of the India-Italy Center for Advanced Research. He is also a member of the International Association for Pattern Recognition and of the Italian Association for Remote Sensing (AIT).

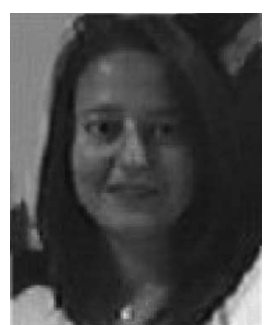

Cristina Tarantino received the laurea degree in physics from the University of Bari, Bari, Italy, in 1998.

She was a Fellow in "high training" with the Institute of Studies on Intelligent System for Automation of the National Research Council, Bari, for three years and has been a Contract Researcher since 2002. She has conducted research work within several national and European projects. Her research interests include remote-sensing image processing and recognition, neural networks and statistical algorithms for classification purposes, partially unsupervised updating of land cover maps, and supervised and unsupervised change detection techniques. 